TRANSACTIONS OF THE

AMERICAN MATHEMATICAL SOCIETY

Volume 365, Number 2, February 2013, Pages 1081-1107

S 0002-9947(2012)05678-9

Article electronically published on August 1, 2012

\title{
FERNIQUE-TYPE INEQUALITIES \\ AND MODULI OF CONTINUITY \\ FOR ANISOTROPIC GAUSSIAN RANDOM FIELDS
}

\author{
MARK M. MEERSCHAERT, WENSHENG WANG, AND YIMIN XIAO \\ ABstract. This paper is concerned with sample path properties of anisotropic \\ Gaussian random fields. We establish Fernique-type inequalities and utilize \\ them to study the global and local moduli of continuity for anisotropic Gauss- \\ ian random fields. Applications to fractional Brownian sheets and to the so- \\ lutions of stochastic partial differential equations are investigated.
}

\section{INTRODUCTION}

Many data sets from various areas such as image processing, hydrology, geostatistics and spatial statistics have anisotropic nature in the sense that they have different geometric and statistical characteristics along different directions, hence fractional Brownian motion is not adequate for modelling such phenomena. Many people have proposed to apply anisotropic Gaussian random fields as more realistic models. See, for example, Davies and Hall [11, Bonami and Estrade [7, Benson et al. 4], and Biermé et al. 6].

Several classes of anisotropic Gaussian random fields have been introduced for theoretical and application purposes. For example, Kamont 15] introduced fractional Brownian sheets and studied some of their regularity properties. Benassi et al. 3] and Bonami and Estrade [7] considered some anisotropic Gaussian random fields with stationary increments. Biermé et al. 6] have constructed a large class of operator self-similar Gaussian or stable random fields with stationary increments. Anisotropic Gaussian random fields also arise naturally in stochastic partial differential equations (see, e.g., Dalang [9], Mueller and Tribe [22], Øksendal and Zhang 24, Nualart 23]) and in studying the most visited sites of symmetric Markov processes (Eisenbaum and Khoshnevisan [12]). Hence it is of importance in both theory and applications to investigate the probabilistic and statistical properties of anisotropic random fields.

Recently, Xiao 32 investigated sample path properties of anisotropic Gaussian random fields under general conditions. Typical examples for Gaussian random

Received by the editors January 22, 2011 and, in revised form, July 27, 2011.

2010 Mathematics Subject Classification. Primary 60G15, 60G17, 60F10, 60F15.

Key words and phrases. Gaussian random field, anisotropy, fractional Brownian sheet, modulus of continuity, law of the iterated logarithm.

The research of the first author was supported by NSF grants DMS-0417869, DMS-0803360 and EAR-0823965.

The research of the second author was supported by NSFC grant 11071076 and NSF grant DMS-0417869.

The research of the third author was supported by NSF grant DMS-0706728.

The second author is the corresponding author for this paper. 
fields covered by his framework are fractional Brownian sheets, operator-scaling Gaussian fields with stationary increments, and the solution to the stochastic heat equation. In the present paper, we are concerned with the global and local moduli of continuity of general anisotropic Gaussian random fields. Our objective is to characterize the anisotropic nature of Gaussian random fields, from an analytic point of view, in terms of their Hurst parameters explicitly.

For this purpose, we first establish Fernique-type inequalities which may be of interest beyond the scope of the present paper. Then we utilize these inequalities to study the global moduli of continuity and the local moduli of continuity (or the law of the iterated logarithm) for anisotropic Gaussian random fields.

Many authors have investigated moduli of continuity of Gaussian random fields. When $W=\left\{W(t), t \in \mathbb{R}_{+}^{N}\right\}$ is an $N$-parameter Brownian sheet, Orey and Pruitt 25] considered increments of $W$ over intervals and points, and established the corresponding global and local moduli of continuity. Benassi et al. 3 proved, among other things, results on the global and local moduli of continuity for a large class of elliptic Gaussian random fields. Ayache and Xiao [2] studied the global moduli of continuity for fractional Brownian sheets by using the wavelet method, and they obtained a sharp upper bound for the global moduli of continuity for points. Wang [29] investigated the global and the local moduli of continuity for fractional Brownian sheets for intervals. As an immediate consequence of our results in the present paper, we give sharp results for the global and local moduli of continuity for fractional Brownian sheets for points.

Throughout this paper, we will use $c$ to denote an unspecified positive and finite constant which may not be the same in each occurrence. More specific constants in Section $i(i=2, \ldots, 6)$ are numbered as $c_{i, 1}, c_{i, 2}, \ldots$.

\section{General assumptions}

The parameter space is $\mathbb{R}^{N}$ or $\mathbb{R}_{+}^{N}=[0, \infty)^{N}$, endowed with the Euclidean norm $\|\cdot\|$. A typical parameter ("time point") is $t=\left(t_{1}, \ldots, t_{N}\right)$, sometimes also written as $\left\langle t_{i}\right\rangle$ or $\langle c\rangle$, if $t_{1}=\cdots=t_{N}=c$. The inner product of $s, t \in \mathbb{R}^{N}$ is denoted by $\langle s, t\rangle$. Given two points $s=\left\langle s_{i}\right\rangle, t=\left\langle t_{i}\right\rangle \in \mathbb{R}_{+}^{N}, s \leq t$ (resp. $s<t$ ) means that $s_{i} \leq t_{i}$ (resp. $\left.s_{i}<t_{i}\right)$ for all $1 \leq i \leq N$. When $s \leq t$, we use $[s, t]$ to denote the $N$-dimensional interval $[s, t]=\times_{i=1}^{N}\left[s_{i}, t_{i}\right]$. For $x \in \mathbb{R}_{+}$, let $\log x=\ln (x \vee e)$, $\log \log x=\ln (\ln (x) \vee e)$.

Let $X=\left\{X(t), t \in \mathbb{R}^{N}\right\}$ be a centered Gaussian random field with values in $\mathbb{R}$. Let $I \subset \mathbb{R}^{N}$ be a fixed compact $N$-dimensional interval, and our goal is to determine the exact uniform and local moduli of continuity of $X(t)$ when $t \in I$. Typically in this paper, we will take $I=[0,1]^{N}$ or $I=[a, 1]^{N}$, where $a \in(0,1)$ is a fixed constant.

Many sample path properties of the Gaussian random field $X$ can be determined by the function

$$
\sigma^{2}(s, t)=\mathbb{E}(X(s)-X(t))^{2}, \quad \forall s, t \in \mathbb{R}^{N} .
$$

As shown by Xiao [32, the following general conditions are useful. Let $H=$ $\left(H_{1}, \ldots, H_{N}\right) \in(0,1]^{N}$ be a fixed vector, and denote

$$
\rho(s, t)=\sum_{j=1}^{N}\left|s_{j}-t_{j}\right|^{H_{j}}, \quad \forall s, t \in \mathbb{R}^{N} .
$$


(A1) There exist positive and finite constants $c_{2,1}$ and $c_{2,2}$ such that

$$
c_{2,1} \rho(s, t)^{2} \leq \sigma^{2}(s, t) \leq c_{2,2} \rho(s, t)
$$

for all $s, t \in I$.

(A2) There exists a constant $c_{2,3}>0$ such that for all integers $n \geq 1$, all $u, t^{1}, \ldots, t^{n} \in I$

$$
\operatorname{Var}\left(X(u) \mid X\left(t^{1}\right), \ldots, X\left(t^{n}\right)\right) \geq c_{2,3} \sum_{j=1}^{N} \min _{0 \leq k \leq n}\left|u_{j}-t_{j}^{k}\right|^{2 H_{j}},
$$

where $t_{j}^{0}=0$ for every $j=1, \ldots, N$.

(A3) There exists a constant $c_{2,4}>0$ such that for all integers $n \geq 1$, and all $u, t^{1}, \ldots, t^{n} \in I$,

$$
\operatorname{Var}\left(X(u) \mid X\left(t^{1}\right), \ldots, X\left(t^{n}\right)\right) \geq c_{2,4} \min _{0 \leq k \leq n} \rho\left(u, t^{k}\right)^{2},
$$

where $t^{0}=0$.

Remark 2.1. The following are some remarks about the above conditions.

- It is helpful to note that $\rho(s, t)$ defined above is a metric on $\mathbb{R}^{N}$. It is more convenient for studying anisotropic random fields than the Euclidean metric.

- Under condition (A1), $X$ has a version which has continuous sample functions on $I$ almost surely. Henceforth we will assume without loss of generality that the Gaussian random field $X$ has continuous sample paths.

- Pitt 26] proved that fractional Brownian motion $X^{\alpha}$ satisfies condition (A3) for all intervals $I \in \mathbb{R}^{N}$ with $H=\langle\alpha\rangle$. Khoshnevisan and Xiao [16] proved that, for every $\varepsilon>0$, the Brownian sheet $W$ satisfies the property (A2) with $H=\langle 1 / 2\rangle$ for all intervals $I \in[\varepsilon, \infty)^{N}$. It has been proved in Ayache and Xiao [2] and in $\mathrm{Wu}$ and Xiao [30] that fractional Brownian sheets satisfy conditions (A1) and (A2) for all intervals $I \subset[\varepsilon, \infty)^{N}$.

- Condition (A3) is listed here mainly for comparison purpose. Condition (A3) implies (A2). It is known that the converse does not even hold for the Brownian sheet; see, e.g., Khoshnevisan and Xiao [16] or Xiao 32]. Roughly speaking, when $H=\langle\alpha\rangle$, the behavior of a Gaussian random field $X$ satisfying conditions (A1) and (A3) is comparable to that of a fractional Brownian motion of index $\alpha$; while a Gaussian random field $X$ satisfying conditions (A1) and (A2) is comparable to that of a fractional Brownian sheet. Hence, in analogy to terminology, for fractional Brownian motion and the Brownian sheet, respectively, condition (A3) will be called the strong local nondeterminism (in metric $\rho$ ) and condition (A2) will be called the sectorial local nondeterminism.

In this paper, we establish the global and local moduli of continuity for Gaussian random fields satisfying conditions (A1) and (A2). The rest of the paper is organized as follows. In Section 3 we state and prove Fernique-type inequalities for anisotropic Gaussian random fields, which will be used in latter sections. The global moduli of continuity are discussed in Section 4 and the local moduli of continuity are investigated in Section 5. Some applications are discussed in Section 6 . 


\section{FERnique-type inequalities}

The aim of this section is to establish Fernique-type inequalities for anisotropic Gaussian random fields, which will be used in latter sections and may be of independent interest. We start with the following lemma, which is a consequence of the results in Fernique [13] and Berman [5].

Lemma 3.1. Suppose that $Y=\left\{Y(t), t \in \mathbb{R}^{N}\right\}$ is a centered Gaussian random field with values in $\mathbb{R}$, and denote

$$
d(s, t):=d_{Y}(s, t)=\left(\mathbb{E}|Y(t)-Y(s)|^{2}\right)^{1 / 2}, \quad s, t \in \mathbb{R}^{N} .
$$

Let $S$ be a closed cube in $\mathbb{R}^{N}$ of edge-length $\delta$ and let $\sigma^{2}=\sup _{t \in S} \mathbb{E}\left(Y(t)^{2}\right)$. For any $h>0, \varepsilon>0$, define

$$
\gamma(\varepsilon)=\sup _{s, t \in S,\|s-t\| \leq \varepsilon} d(s, t)
$$

and

$$
Q(h)=(2+\sqrt{2}) \int_{1}^{\infty} \gamma\left(h 2^{-y^{2}}\right) d y .
$$

Then for all $x>0$ which satisfy $x \geq(1+4 N \log 2)^{1 / 2}\left(\sigma+x^{-1}\right)$,

$$
\mathbb{P}\left\{\sup _{t \in S}|Y(t)|>x\right\} \leq 2^{2 N+2}\left(\frac{\delta}{Q^{-1}(1 / x)}+1\right)^{N} \frac{\sigma+x^{-1}}{x} \exp \left(-\frac{x^{2}}{2\left(\sigma+x^{-1}\right)^{2}}\right),
$$

where $Q^{-1}(x)=\sup \{y: Q(y) \leq x\}$. Particularly, from (3.1) it follows that for any $\varepsilon>0$ there exist positive constants $x_{0}=x_{0}(\varepsilon, \sigma)$ and $c_{3,1}=c_{3,1}(\varepsilon, \sigma, N)$ such that for any $x \geq x_{0}$,

$$
\mathbb{P}\left\{\sup _{t \in S}|Y(t)|>x\right\} \leq c_{3,1}\left(\frac{\delta}{Q^{-1}(1 / x)}+1\right)^{N} \exp \left(-\frac{x^{2}}{(2+\varepsilon) \sigma^{2}}\right) .
$$

Proof. For every $h \in(0, \delta], S$ can be covered by $(\lfloor\delta / h\rfloor+1)^{N}$ closed subcubes $\left\{S_{i}\right\}$ of side-length $h$, where $\lfloor u\rfloor$ denotes the largest integer $\leq u$. Hence

$$
\mathbb{P}\left\{\sup _{t \in S}|Y(t)|>x\right\} \leq\left(\left\lfloor\frac{\delta}{h}\right\rfloor+1\right)^{N} \max _{i} \mathbb{P}\left\{\sup _{t \in S_{i}}|Y(t)|>x\right\} .
$$

Take $h=Q^{-1}(1 / x) \wedge \delta$. It follows from the Fernique inequality (with $p=2$ ) in Section 4.1.3 of Fernique [13] or (4.2) in Berman [5] that for every subcube $S_{i}$, we have

$$
\mathbb{P}\left\{\sup _{t \in S_{i}}|Y(t)|>x\right\} \leq 5 \sqrt{2 \pi} 2^{2 N-1} \Psi\left(\frac{x}{\sigma+Q(h)}\right)
$$

for all $x \geq(1+4 N \log 2)^{1 / 2}(\sigma+Q(h))$, where $\Psi(u)=\mathbb{P}\{N(0,1)>u\}$ is the tail probability of a standard normal random variable. In deriving (3.4), we have also used the fact that $\Psi(u)$ is decreasing.

Notice that $Q(h) \leq x^{-1}$ and $\Psi(u) \leq(2 \pi)^{-\frac{1}{2}} u^{-1} e^{-\frac{u^{2}}{2}}$ for all $u>0$, we can write the inequality (3.4) as

$$
\mathbb{P}\left\{\sup _{t \in S_{i}}|Y(t)|>x\right\} \leq 52^{2 N-1} \frac{\sigma+x^{-1}}{x} \exp \left(-\frac{x^{2}}{2\left(\sigma+x^{-1}\right)^{2}}\right),
$$

which, together with (3.3), yields (3.1) and thus Lemma 3.1 . 
For the next lemma, we need the following notation. For every $t \in \mathbb{R}^{N}, x \in \mathbb{R}$ and $\ell=1, \ldots, N$, we denote by $\left(\widehat{t}_{\ell}, x\right)$ the vector in $\mathbb{R}^{N}$ obtained from $t$ by replacing its $\ell$ th coordinate $t_{\ell}$ by $x$. For example, $\left(\widehat{t}_{N}, x\right)=\left(t_{1}, \ldots, t_{N-1}, x\right)$.

Lemma 3.2. Let $X=\left\{X(t), t \in \mathbb{R}^{N}\right\}$ be a centered Gaussian random field with values in $\mathbb{R}$ and satisfy the upper bound in condition (A1) with $I=[0,1]^{N}$. Then for any $\varepsilon>0$, there exist positive constants $u_{0}=u_{0}\left(\varepsilon, c_{2,2}\right)$ and $c_{3,2}=$ $c_{3,2}\left(\varepsilon, c_{2,2}, H, N\right)$ such that for all $x \in[0,1], 0<y \leq z \leq 1$ that satisfy $[x, x+y] \subset$ $[0,1]$, all $u \geq u_{0}$ and $1 \leq \ell \leq N$

$$
\mathbb{P}\left\{\sup _{t \in[0,1]^{N}}\left|X\left(\widehat{t}_{\ell}, x+y\right)-X\left(\widehat{t}_{\ell}, x\right)\right| \geq u z^{H_{\ell}}\right\} \leq c_{3,2} z^{-\frac{(N-1) H_{\ell}}{\min \left\{H_{i}\right\}}} u^{\frac{N-1}{\min \left\{H_{i}\right\}}} e^{-\frac{u^{2}}{(2+\varepsilon) c_{2,2}}} .
$$

Here and in the sequel the minimum of $H_{i}$ is taken over all $1 \leq i \leq N$.

Proof. For simplicity of notation, we only prove (3.6) for $\ell=N$ and write $\left(\widehat{t}_{N}, x\right)$ as $(t, x)$, where $t \in[0,1]^{N-1}$ or more generally $t \in \mathbb{R}^{N-1}$. For any $x \in[0,1]$ and $0<y \leq z$, we consider the Gaussian process $Y=\left\{Y(t), t \in \mathbb{R}^{N-1}\right\}$ defined by

$$
Y(t)=\frac{X(t, x+y)-X(t, x)}{z^{H_{N}}}, \quad \forall t \in \mathbb{R}^{N-1} .
$$

We now show (3.6) by applying Lemma 3.1 to $Y$ with $S=[0,1]^{N-1}$.

By the Minkowski inequality and condition (A1), we have

$$
\begin{aligned}
d(s, t) & \leq \frac{1}{z^{H_{N}}}\left[\mathbb{E}(X(t, x+y)-X(s, x+y))^{2}+\mathbb{E}(X(t, x)-X(s, x))^{2}\right]^{1 / 2} \\
& \leq \frac{2 c_{2,2}^{1 / 2}}{z^{H_{N}}} \sum_{j=1}^{N-1}\left|s_{j}-t_{j}\right|^{H_{j}}
\end{aligned}
$$

for all $s, t \in S$. By Jensen's inequality we derive

$$
\begin{aligned}
\sum_{j=1}^{N-1}\left|t_{j}-s_{j}\right|^{2 H_{j}} & \leq(N-1)^{1-\min \left\{H_{i}\right\}}\left(\sum_{j=1}^{N-1}\left|t_{j}-s_{j}\right|^{2 H_{j} / \min \left\{H_{i}\right\}}\right)^{\min \left\{H_{i}\right\}} \\
& \leq(N-1)^{1-\min \left\{H_{i}\right\}}\left(\sum_{j=1}^{N-1}\left|t_{j}-s_{j}\right|^{2}\right)^{\min \left\{H_{i}\right\}} \\
& =(N-1)^{1-\min \left\{H_{i}\right\}}\|t-s\|^{2 \min \left\{H_{i}\right\}} .
\end{aligned}
$$

Thus

$$
d(s, t) \leq \frac{2 c_{2,2}^{1 / 2}}{z^{H_{N}}}(N-1)^{\left(1-\min \left\{H_{i}\right\}\right) / 2}\|t-s\|^{\min \left\{H_{i}\right\}} .
$$

It follows that

$$
\gamma(\varepsilon)=\sup _{s, t \in S,\|s-t\| \leq \varepsilon} d(s, t) \leq \frac{2 c_{2,2}^{1 / 2}}{z^{H_{N}}}(N-1)^{\left(1-\min \left\{H_{i}\right\}\right) / 2} \varepsilon^{\min \left\{H_{i}\right\}} .
$$

This implies that

$$
Q(h) \leq c h^{\min \left\{H_{i}\right\}} / z^{H_{N}},
$$

and the inverse function of $Q$ satisfies

$$
Q^{-1}(r) \geq c r^{1 / \min \left\{H_{i}\right\}} z^{H_{N} / \min \left\{H_{i}\right\}} .
$$


Since $\sigma^{2}=\sup _{t \in S} \mathbb{E}\left(Y(t)^{2}\right) \leq c_{2,2}$ by condition (A1), we use (3.2) to derive that for any $\varepsilon>0$, there exists a positive constant $u_{0}=u_{0}\left(\varepsilon, c_{2,2}\right)$ such that for all $u \geq u_{0}$,

$$
\begin{aligned}
& \mathbb{P}\left\{\sup _{t \in[0,1]^{N-1}}|X(t, x+y)-X(t, x)| \geq u z^{H_{N}}\right\} \\
& \leq c u^{(N-1) / \min \left\{H_{i}\right\}} z^{-(N-1) H_{N} / \min \left\{H_{i}\right\}} e^{-\frac{u^{2}}{(2+\varepsilon) c_{2,2}}} .
\end{aligned}
$$

This yields (3.6) for $\ell=N$.

The following is the main result of this section, which is a generalization of Lemma 2.1 of Csáki et al. 8 for Gaussian random fields.

Proposition 3.3. Let $\left\{X(t), t \in \mathbb{R}^{N}\right\}$ be a centered Gaussian random field in $\mathbb{R}$ satisfying the upper bound in condition (A1) for $I=[0,1]^{N}$. For any $\tau>0$ and any $\varepsilon>0$ there exist positive constants $u^{*}=u^{*}\left(\varepsilon, c_{2,2}\right)$ and $c_{3,3}=c\left(\tau, \varepsilon, c_{2,2}, H, N\right)$ such that

$$
\begin{aligned}
& \mathbb{P}\left\{\sup _{\left\langle x_{i}\right\rangle \leq t \leq\left\langle x_{i}+T_{i}\right\rangle\langle 0\rangle \leq s \leq\left\langle a_{i}\right\rangle} \sup _{\langle}|X(t+s)-X(t)| \geq((1+\tau) u+\tau) \sum_{j=1}^{N} a_{j}^{H_{j}}\right\} \\
& \leq c_{3,3}\left[\sum_{j=1}^{N}\left(\frac{T_{j}}{a_{j}}+1\right) \cdot\left(\frac{1}{a_{j}}\right)^{\frac{(N-1) H_{j}}{\min \left\{H_{i}\right\}}}\right]\left(u^{\frac{N-1}{\min \left\{H_{i}\right\}}}+1\right) e^{-\frac{u^{2}}{(2+\varepsilon) c_{2,2}}}
\end{aligned}
$$

for all $u \geq u^{*}, x_{i}, T_{i} \in[0,1]$, and $a_{i} \in(0,1](i=1, \ldots, N)$ which satisfy $\left[\left\langle x_{i}\right\rangle,\left\langle x_{i}+T_{i}+a_{i}\right\rangle\right] \subset[0,1]^{N}$.

Proof. We prove (3.7) by using induction on $N$. If $N=1,(3.7)$ is an immediate consequence of Lemma 2.1 of Csáki et al. [8].

Now we consider the case $N \geq 2$. It is easy to see that for any $s, t \in \mathbb{R}^{N}$,

$$
\begin{array}{r}
X(t+s)-X(t)=\sum_{j=1}^{N}\left(X\left(t_{1}, \ldots, t_{j-1}, t_{j}+s_{j}, t_{j+1}+s_{j+1}, \ldots, t_{N}+s_{N}\right)\right. \\
\left.-X\left(t_{1}, \ldots, t_{j-1}, t_{j}, t_{j+1}+s_{j+1}, \ldots, t_{N}+s_{N}\right)\right)
\end{array}
$$

with the convention that $X\left(t_{1}, \ldots, t_{j-1}, t_{j}+s_{j}, t_{j+1}+s_{j+1}, \ldots, t_{N}+s_{N}\right)=X(t+s)$ if $j=1$ and $X\left(t_{1}, \ldots, t_{j-1}, t_{j}, t_{j+1}+s_{j+1}, \ldots, t_{N}+s_{N}\right)=X(t)$ if $j=N$. By (3.8), we have for each $N \geq 2$

$$
\begin{aligned}
& \sup _{\left\langle x_{i}\right\rangle \leq t \leq\left\langle x_{i}+T_{i}\right\rangle} \sup _{\langle 0\rangle \leq s \leq\left\langle a_{i}\right\rangle}|X(t+s)-X(t)| \\
& \quad \leq \sum_{j=1}^{N} \sup _{t \in[0,1]^{N-1}} \sup _{x_{j} \leq t_{j} \leq x_{j}+T_{j}} \sup _{0 \leq s_{j} \leq a_{j}}\left|X\left(t, t_{j}+s_{j}\right)-X\left(t, t_{j}\right)\right| .
\end{aligned}
$$

Here and in the rest of the proof, for $t \in \mathbb{R}^{N-1}$, we write $\left(t, t_{j}\right) \in \mathbb{R}^{N}$ for the point whose $j$ th coordinate is $t_{j}$ and so $\left(t, t_{j}\right)=\left(t_{1}, \ldots, t_{N}\right)$. 
Thus for any $\tau>0$ and $u>0$,

$$
\begin{aligned}
& \mathbb{P}\left\{\sup _{\left\langle x_{i}\right\rangle \leq t \leq\left\langle x_{i}+T_{i}\right\rangle} \sup _{\langle 0\rangle \leq s \leq\left\langle a_{i}\right\rangle}|X(t+s)-X(t)| \geq((1+\tau) u+\tau) \sum_{j=1}^{N} a_{j}^{H_{j}}\right\} \\
& \leq \sum_{j=1}^{N} \mathbb{P}\left\{\sup _{t \in[0,1]^{N-1}} \sup _{x_{j} \leq t_{j} \leq x_{j}+T_{j}} \sup _{0 \leq s_{j} \leq a_{j}}\left|X\left(t, t_{j}+s_{j}\right)-X\left(t, t_{j}\right)\right| \geq((1+\tau) u+\tau) a_{j}^{H_{j}}\right\} \\
& =: \sum_{j=1}^{N} P_{j} .
\end{aligned}
$$

Let $1 \leq j \leq N$ be fixed. We follow the proof of Lemma 2.1 of Csáki et al. 8 to estimate $P_{j}(1 \leq j \leq N)$ by using a chaining argument. For any positive real number $r$ and $n \geq 3$, denote $(r)_{n}=a_{j}\left\lfloor r \frac{2^{n}}{a_{j}}\right\rfloor / 2^{n}$, where $\lfloor x\rfloor$ is the largest integer $\leq x$. Clearly $(r)_{n} \rightarrow r$ as $n \rightarrow \infty$.

Let $k \geq 3$ be a fixed integer whose value will be specified later. By the triangle inequality, we have

$$
\begin{aligned}
\mid X( & \left.t, t_{j}+s_{j}\right)-X\left(t, t_{j}\right) \mid \\
\leq & \left|X\left(t,\left(t_{j}+s_{j}\right)_{k}\right)-X\left(t,\left(t_{j}\right)_{k}\right)\right| \\
& \left.+\left|X\left(t, t_{j}+s_{j}\right)-X\left(t,\left(t_{j}+s_{j}\right)_{k}\right)\right|+\mid X\left(t, t_{j}\right)-X\left(t, t_{j}\right)_{k}\right) \mid \\
\leq & \left|X\left(t,\left(t_{j}+s_{j}\right)_{k}\right)-X\left(t,\left(t_{j}\right)_{k}\right)\right| \\
& +\sum_{l=0}^{\infty}\left|X\left(t,\left(t_{j}+s_{j}\right)_{k+l+1}\right)-X\left(t,\left(t_{j}+s_{j}\right)_{k+l}\right)\right| \\
& +\sum_{l=0}^{\infty}\left|X\left(t,\left(t_{j}\right)_{k+l+1}\right)-X\left(t,\left(t_{j}\right)_{k+l}\right)\right| .
\end{aligned}
$$

In order to use the above inequality to estimate $P_{j}$, we make some preparation first. For $l \geq 0$, put

$$
u_{l}^{2}=u^{2}+c_{2,2}\left(2+\frac{2(N-1) H_{j}}{\min \left\{H_{i}\right\}}+\frac{N-1}{\min \left\{H_{i}\right\}}\right)(k+l+1) .
$$

Then

$$
u_{l} \leq u+c_{2,2}^{1 / 2}\left(2+\frac{2(N-1) H_{j}}{\min \left\{H_{i}\right\}}+\frac{N-1}{\min \left\{H_{i}\right\}}\right)^{1 / 2}(k+l+1)^{1 / 2} .
$$


It follows that

$$
\begin{aligned}
& u a_{j}^{H_{j}}+u\left(\frac{2 a_{j}}{2^{k}}\right)^{H_{j}}+2 \sum_{l=0}^{\infty} u_{l}\left(\frac{a_{j}}{2^{k+l}}\right)^{H_{j}} \\
& \leq u a_{j}^{H_{j}}+u\left[\left(\frac{2 a_{j}}{2^{k}}\right)^{H_{j}}+\sum_{l=0}^{\infty}\left(\frac{a_{j}}{2^{k+l}}\right)^{H_{j}}\right] \\
& \quad+c_{2,2}^{1 / 2}\left(2+\frac{2(N-1) H_{j}}{\min \left\{H_{i}\right\}}+\frac{N-1}{\min \left\{H_{i}\right\}}\right)^{1 / 2} \sum_{l=0}^{\infty}(k+l+1)^{1 / 2}\left(\frac{a_{j}}{2^{k+l}}\right)^{H_{j}} \\
& =u a_{j}^{H_{j}}+u a_{j}^{H_{j}} \frac{2^{H_{j}}+\left(1-2^{-H_{j}}\right)^{-1}}{2^{k H_{j}}} \\
& \quad+c_{2,2}^{1 / 2} a_{j}^{H_{j}}\left(2+\frac{2(N-1) H_{j}}{\min \left\{H_{i}\right\}}+\frac{N-1}{\min \left\{H_{i}\right\}}\right)^{1 / 2} \sum_{l=0}^{\infty} \frac{(k+l+1)^{1 / 2}}{2^{H_{j}(k+l)}} \\
& \leq u a_{j}^{H_{j}}+\tau u a_{j}^{H_{j}}+\tau a_{j}^{H_{j}}=((1+\tau) u+\tau) a_{j}^{H_{j}} .
\end{aligned}
$$

In deriving the last inequality, we have chosen $k$ large enough such that

$$
\frac{2^{H_{j}}+\left(1-2^{-H_{j}}\right)^{-1}}{2^{k H_{j}}} \leq \tau
$$

and

$$
c_{2,2}^{1 / 2}\left(2+\frac{2(N-1) H_{j}}{\min \left\{H_{i}\right\}}+\frac{N-1}{\min \left\{H_{i}\right\}}\right)^{1 / 2} \sum_{l=k}^{\infty} \frac{(l+1)^{1 / 2}}{2^{H_{j} l}} \leq \tau .
$$

It follows from (3.10) and (3.11) that

$$
\begin{aligned}
P_{j} \leq & \mathbb{P}\left\{\sup _{t \in[0,1]^{N-1}} \sup _{x_{j} \leq t_{j} \leq x_{j}+T_{j}} \sup _{0 \leq s_{j} \leq a_{j}}\right. \\
& \left.\times\left|X\left(t,\left(t_{j}+s_{j}\right)_{k}\right)-X\left(t,\left(t_{j}\right)_{k}\right)\right| \geq\left(1+2^{-k+1}\right) u a_{j}^{H_{j}}\right\} \\
+ & \sum_{l=0}^{\infty} \mathbb{P}\left\{\sup _{t \in[0,1]^{N-1}} \sup _{x_{j} \leq t_{j} \leq x_{j}+T_{j}} \sup _{0 \leq s_{j} \leq a_{j}}\right. \\
& +\sum_{l=0}^{\infty} \mathbb{P}\left\{\begin{array}{l}
\sup _{t \in[0,1]^{N-1}} x_{j} \leq t_{j} \leq x_{j}+T_{j} \\
\left.\sup _{k}\left(t,\left(t_{j}+s_{j}\right)_{k+l+1}\right)-X\left(t,\left(t_{j}+s_{j}\right)_{k+l}\right) \mid \geq u_{l}\left(\frac{a_{j}}{2^{k+l}}\right)^{H_{j}}\right\}
\end{array}\right. \\
& \left.\times\left|X\left(t,\left(t_{j}\right)_{k+l+1}\right)-X\left(t,\left(t_{j}\right)_{k+l}\right)\right| \geq u_{l}\left(\frac{a_{j}}{2^{k+l}}\right)^{H_{j}}\right\} \\
= & P_{j, 1}+P_{j, 2}+P_{j, 3} .
\end{aligned}
$$


We estimate the terms $P_{j, 1}, P_{j, 2}$, and $P_{j, 3}$ separately. In order to estimate $P_{j, 1}$, we use the triangle inequality again to write

$$
\begin{aligned}
\sup _{t \in[0,1]^{N-1}} \sup _{x_{j} \leq t_{j} \leq x_{j}+T_{j}} \sup _{0 \leq s_{j} \leq a_{j}} \\
\quad \times\left|X\left(t,\left(t_{j}+s_{j}\right)_{k}\right)-X\left(t,\left(t_{j}\right)_{k}\right)\right| \\
\leq \sup _{t \in[0,1]^{N-1}} \sup _{x_{j} \leq t_{j} \leq x_{j}+T_{j}} \sup _{0 \leq s_{j} \leq a_{j}\left(1-2^{-k}\right)} \\
\quad \times\left|X\left(t,\left(t_{j}+s_{j}\right)_{k}\right)-X\left(t,\left(t_{j}\right)_{k}\right)\right| \\
+\sup _{t \in[0,1]^{N-1}} \sup _{x_{j} \leq t_{j} \leq x_{j}+T_{j}} \sup _{a_{j}\left(1-2^{-k}\right) \leq s_{j} \leq a_{j}} \\
\quad \times\left|X\left(t,\left(t_{j}+s_{j}\right)_{k}\right)-X\left(t,\left(t_{j}+a_{j}\left(1-2^{-k}\right)\right)_{k}\right)\right| .
\end{aligned}
$$

Since

$$
\sup _{0 \leq t_{j} \leq T_{j}} \sup _{0 \leq s_{j} \leq a_{j}\left(1-2^{-k}\right)}\left|\left(t_{j}+s_{j}\right)_{k}-\left(t_{j}\right)_{k}\right| \leq a_{j},
$$

and there are at most $2^{k}\left(\frac{T_{j}}{a_{j}}+1\right)$ different points $\left(t_{j}\right)_{k}$ and at most $2^{k}$ different $\left(t_{j}+s_{j}\right)_{k}$, we derive from Lemma 3.2 with $z=a_{j}$ that for each $u \geq u^{*}$,

$$
\begin{gathered}
\mathbb{P}\left\{\sup _{t \in[0,1]^{N-1}} \sup _{x_{j} \leq t_{j} \leq x_{j}+T_{j}} \sup _{0 \leq s_{j} \leq a_{j}\left(1-2^{-k}\right)}\left|X\left(t,\left(t_{j}+s_{j}\right)_{k}\right)-X\left(t,\left(t_{j}\right)_{k}\right)\right| \geq u a_{j}^{H_{j}}\right\} \\
\leq c_{3,2} 2^{2 k}\left(\frac{T_{j}}{a_{j}}+1\right) \cdot\left(\frac{1}{a_{j}}\right)^{\frac{(N-1) H_{j}}{\min \left\{H_{i}\right\}}} u^{\frac{N-1}{\min \left\{H_{i}\right\}}} \exp \left(-\frac{u^{2}}{(2+\varepsilon) c_{2,2}}\right) .
\end{gathered}
$$

Similarly, because

$$
\sup _{x_{j} \leq t_{j} \leq x_{j}+T_{j}} \sup _{a_{j}\left(1-2^{-k}\right) \leq s_{j} \leq a_{j}}\left|\left(t_{j}+s_{j}\right)_{k}-\left(t_{j}+a_{j}\left(1-2^{-k}\right)\right)_{k}\right| \leq 2 a_{j} \cdot 2^{-k},
$$

we derive from Lemma 3.2 that for each $u \geq u^{*}$,

$$
\begin{aligned}
& \mathbb{P}\left\{\sup _{t \in[0,1]^{N-1}} \sup _{x_{j} \leq t_{j} \leq x_{j}+T_{j}} \sup _{a_{j}\left(1-2^{-k}\right) \leq s_{j} \leq a_{j}}\right. \\
& \left.\quad \times\left|X\left(t,\left(t_{j}+s_{j}\right)_{k}\right)-X\left(t,\left(t_{j}+a_{j}\left(1-2^{-k}\right)\right)_{k}\right)\right| \geq u\left(2^{-k+1} a_{j}\right)^{H_{j}}\right\} \\
& \quad \leq c_{3,2} 2^{k}\left(\frac{T_{j}}{a_{j}}+1\right) \cdot\left(\frac{2^{k+1}}{a_{j}}\right)^{\frac{(N-1) H_{j}}{\min \left\{H_{i}\right\}}} u^{\frac{N-1}{\min \left\{H_{i}\right\}}} \exp \left(-\frac{u^{2}}{(2+\varepsilon) c_{2,2}}\right) .
\end{aligned}
$$

Combining (3.12)-(3.14), we obtain

$$
P_{j, 1} \leq c_{3,4}\left(\frac{T_{j}}{a_{j}}+1\right) \cdot\left(\frac{1}{a_{j}}\right)^{\frac{(N-1) H_{j}}{\min \left\{H_{i}\right\}}} u^{\frac{N-1}{\min \left\{H_{i}\right\}}} \exp \left(-\frac{u^{2}}{(2+\varepsilon) c_{2,2}}\right) .
$$

In the same way, noting that

$$
\sup _{x_{j} \leq t_{j} \leq x_{j}+T_{j}} \sup _{0 \leq s_{j} \leq a_{j}}\left|\left(t_{j}+s_{j}\right)_{k+l+1}-\left(t_{j}+s_{j}\right)_{k+l}\right| \leq a_{j} \cdot 2^{-(k+l)},
$$


we apply Lemma 3.2 to derive

$$
\begin{aligned}
& \mathbb{P}\left\{\sup _{t \in[0,1]^{N-1}} \sup _{x_{j} \leq t_{j} \leq x_{j}+T_{j}} \sup _{0 \leq s_{j} \leq a_{j}}\right. \\
& \left.\quad \times\left|X\left(t,\left(t_{j}+s_{j}\right)_{k+l+1}\right)-X\left(t,\left(t_{j}+s_{j}\right)_{k+l}\right)\right| \geq u_{l}\left(a_{j} 2^{-(k+l)}\right)^{H_{j}}\right\} \\
& \leq c_{3,2} 2^{k+l+1}\left(\frac{T_{j}}{a_{j}}+1\right) \cdot\left(\frac{2^{k+l}}{a_{j}}\right)^{\frac{(N-1) H_{j}}{\min \left\{H_{i}\right\}}} u_{l}^{\frac{N-1}{\min \left\{H_{i}\right\}}} \exp \left(-\frac{u_{l}^{2}}{(2+\varepsilon) c_{2,2}}\right)
\end{aligned}
$$

and

$$
\begin{aligned}
& \mathbb{P}\left\{\sup _{t \in[0,1]^{N-1}} \sup _{x_{j} \leq t_{j} \leq x_{j}+T_{j}}\left|X\left(t,\left(t_{j}\right)_{k+l+1}\right)-X\left(t,\left(t_{j}\right)_{k+l}\right)\right| \geq u_{l}\left(a_{j} 2^{-(k+l)}\right)^{H_{j}}\right\} \\
& \leq c_{3,2} 2^{k+l+1}\left(\frac{T_{j}}{a_{j}}+1\right) \cdot\left(\frac{2^{k+l}}{a_{j}}\right)^{\frac{(N-1) H_{j}}{\min \left\{H_{i}\right\}}} u_{l}^{\frac{N-1}{\min \left\{H_{i}\right\}}} \exp \left(-\frac{u_{l}^{2}}{(2+\varepsilon) c_{2,2}}\right) .
\end{aligned}
$$

By the definition of $u_{l}$, we have

$$
\begin{aligned}
& u_{l}^{\frac{N-1}{\min \left\{H_{i}\right\}}} \leq 2^{\frac{N-1}{\min \left\{H_{i}\right\}}-1} \\
& \quad \times\left[u^{\frac{N-1}{\min \left\{H_{i}\right\}}} c_{2,2}^{\frac{N-1}{2 \min \left\{H_{i}\right\}}}\left(2+\frac{(N-1)\left(2 H_{j}+1\right)}{\min \left\{H_{i}\right\}}\right)^{\frac{N-1}{2 \min \left\{H_{i}\right\}}}(k+l+1)^{\frac{N-1}{2 \min \left\{H_{i}\right\}}}\right]
\end{aligned}
$$

and

$$
\begin{aligned}
& \sum_{l=0}^{\infty} 2^{\left(1+\frac{(N-1) H_{j}}{\min \left\{H_{i}\right\}}\right)(k+l+1)}(k+l+1)^{\frac{N-1}{2 \min \left\{H_{i}\right\}}} \exp \left(-\frac{u_{l}^{2}}{(2+\varepsilon) c_{2,2}}\right) \\
& \leq \sum_{l=0}^{\infty} 2^{\left(1+\frac{(N-1) H_{j}}{\min \left\{H_{i}\right\}}+\frac{N-1}{2 \min \left\{H_{i}\right\}}\right)(k+l+1)} \exp \left(-\frac{u_{l}^{2}}{(2+\varepsilon) c_{2,2}}\right) \\
& =\sum_{l=0}^{\infty} 2^{\left(1+\frac{(N-1) H_{j}}{\min \left\{H_{i}\right\}}+\frac{N-1}{2 \min \left\{H_{i}\right\}}\right)(k+l+1)} \\
& \quad \times \exp \left(-\left(1+\frac{(N-1) H_{j}}{\min \left\{H_{i}\right\}}+\frac{N-1}{2 \min \left\{H_{i}\right\}}\right)(k+l+1)\right) \exp \left(-\frac{u^{2}}{(2+\varepsilon) c_{2,2}}\right) \\
& \leq \exp \left(-\frac{u^{2}}{(2+\varepsilon) c_{2,2}}\right) .
\end{aligned}
$$

Hence, (3.16) and (3.17) yield

$$
P_{j, 2}+P_{j, 3} \leq c_{3,5}\left(\frac{T_{j}}{a_{j}}+1\right) \cdot\left(\frac{1}{a_{j}}\right)^{\frac{(N-1) H_{j}}{\min \left\{H_{i}\right\}}}\left(u^{\frac{N-1}{\min \left\{H_{i}\right\}}}+1\right) \exp \left(-\frac{u^{2}}{(2+\varepsilon) c_{2,2}}\right) .
$$

Combining the above inequality with (3.15) shows that for each $1 \leq j \leq N$

$$
P_{j} \leq c_{3,5}\left(\frac{T_{j}}{a_{j}}+1\right) \cdot\left(\frac{1}{a_{j}}\right)^{\frac{(N-1) H_{j}}{\min \left\{H_{i}\right\}}}\left(u^{\frac{N-1}{\min \left\{H_{i}\right\}}}+1\right) \exp \left(-\frac{u^{2}}{(2+\varepsilon) c_{2,2}}\right) .
$$

This, together with (3.9), implies (3.7). The proof is now completed. 


\section{Global modulus of continuity}

In this section we investigate the global modulus of continuity for anisotropic Gaussian random fields. As a consequence of our result, we establish a sharp result for the global modulus of continuity of fractional Brownian sheets for points, which extend Theorem 1 of Ayache and Xiao [2] and Theorem 3.2 of Wang [29]; see Section 6 below.

Theorem 4.1. Let $\left\{X(t), t \in \mathbb{R}^{N}\right\}$ be a centered Gaussian random field with values in $\mathbb{R}$, and let it satisfy conditions (A1) and (A2). Put

$$
\beta(s, t)=\sigma(s, t) \sqrt{\log \left(1+\sigma(s, t)^{-1}\right)}, \quad s, t \in I .
$$

Then

$$
\lim _{\varepsilon \rightarrow 0+} \sup _{s, t \in I, \sigma(s, t) \leq \varepsilon} \frac{|X(t)-X(s)|}{\beta(s, t)}=\kappa_{1} \quad \text { a.s. },
$$

where $\kappa_{1}$ is a positive constant satisfying

$$
\sqrt{\frac{2 c_{2,3}}{c_{2,2} \min \left\{H_{i}\right\}}} \leq \kappa_{1} \leq \sqrt{\frac{2 N^{3} c_{2,2}}{c_{2,1} \min \left\{H_{i}\right\}}} .
$$

Proof. For simplicity of notation, we assume $I=[a, 1]^{N}$, where $a \in[0,1)$ is a constant. For any $\varepsilon>0$, put

$$
J(\varepsilon)=\sup _{s, t \in I, \sigma(s, t) \leq \varepsilon} \frac{|X(t)-X(s)|}{\beta(s, t)},
$$

then $\varepsilon \mapsto J(\varepsilon)$ is non-decreasing. Hence the limit in the left-hand side of (4.2) exists almost surely. We claim that

$$
\lim _{\varepsilon \rightarrow 0+} J(\varepsilon) \leq c_{4,2} \quad \text { a.s. }
$$

and

$$
\lim _{\varepsilon \rightarrow 0+} J(\varepsilon) \geq c_{4,1} \quad \text { a.s. }
$$

where

$$
c_{4,1}=\sqrt{\frac{2 c_{2,3}}{c_{2,2} \min \left\{H_{i}\right\}}} \quad \text { and } \quad c_{4,2}=\sqrt{\frac{2 N^{3} c_{2,2}}{c_{2,1} \min \left\{H_{i}\right\}}} .
$$

Before proving (4.4) and (4.5), let us notice that (4.4) and the proof of Lemma 7.1.1 in Marcus and Rosen [20] imply (4.2) and the constant $\kappa_{1} \in\left[c_{4,1}, c_{4,2}\right]$.

Hence, it only remains to verify (4.4) and (4.5). We show (4.4) first. Proofs of (4.4) with a generic constant by using general Gaussian principles, such as majorizing measure or isoperimetric inequality, are available; see, e.g., Marcus and Rosen [20, Chapter 7] or Xiao [32. Here we apply Proposition 3.3 to provide a more careful deviation to connect this constant with the constants in (A1). Since the function $x \mapsto x \sqrt{\log (1+1 / x)}$ is increasing for $x \in(0,1)$ small and

$$
\sigma(s, t)^{2} \geq c_{2,1} \max \left\{\left|t_{j}-s_{j}\right|^{2 H_{j}}\right\},
$$

we have

$$
\beta(s, t) \geq c_{2,1}^{1 / 2}\left|t_{j}-s_{j}\right|^{H_{j}} \sqrt{\log \left(1+c_{2,1}^{-1 / 2}\left|t_{j}-s_{j}\right|^{-H_{j}}\right)}
$$


for every $1 \leq j \leq N$. Thus by (3.8) we have

$$
\begin{aligned}
& J(\varepsilon) \leq \sum_{j=1}^{N} \sup _{\substack{t \in[a, 1] \\
\text { s-1, s, } s_{j}, t_{j} \in[a, 1] \\
\sigma(s, t) \leq \varepsilon}} \frac{\left|X\left(t, t_{j}\right)-X\left(t, s_{j}\right)\right|}{\beta(s, t)} \\
& \leq \sum_{j=1}^{N} \sup _{\substack{t \in[a, 1] N-s_{j}, s_{j}, t_{j} \in[a, 1] \\
c_{2}^{1 / 2}\left|t_{j}-s_{j}\right|^{H_{j}<\varepsilon}}} \frac{\left|X\left(t, t_{j}\right)-X\left(t, s_{j}\right)\right|}{\beta(s, t)} \\
& \leq \sum_{j=1}^{N} \sup _{\substack{t \in[a, 1] \\
c_{2,1}^{N-1}\left|t_{j}-s_{j}, t_{j}\right|^{1} \in[a, 1]}} \frac{\left|X\left(t, t_{j}\right)-X\left(t, s_{j}\right)\right|}{c_{2,1}^{1 / 2}\left|t_{j}-s_{j}\right|^{H_{j}} \sqrt{\log \left(1+c_{2,1}^{-1 / 2}\left|t_{j}-s_{j}\right|^{-H_{j}}\right)}} \\
& =: \sum_{j=1}^{N} J_{j}(\varepsilon) \text {. }
\end{aligned}
$$

We now show that for each $1 \leq j \leq N$,

$$
\limsup _{\varepsilon \rightarrow 0+} J_{j}(\varepsilon) \leq \frac{c_{4,2}}{N} \quad \text { a.s. }
$$

For every $1 \leq j \leq N$ and $\mu>0$, define the event

$$
E_{j}(l, k, n)=\left\{\sup \frac{\left|X\left(t, t_{j}\right)-X\left(t, s_{j}\right)\right|}{c_{2,1}^{1 / 2}\left|t_{j}-s_{j}\right|^{H_{j}} \sqrt{\log \left(1+c_{2,1}^{-1 / 2}\left|t_{j}-s_{j}\right|^{-H_{j}}\right)}} \geq \frac{(1+\mu) c_{4,2}}{N}\right\},
$$

where the supremum is taken over $t \in[a, 1]^{N-1}$ and all $s_{j}, t_{j}$ which satisfy

$$
\frac{l-1}{2^{n}} \leq s_{j}<\frac{l}{2^{n}}, \quad \frac{l+k}{2^{n}} \leq t_{j}<\frac{l+k+1}{2^{n}} .
$$

Let

$$
A=c_{2,1}^{1 / 2} k^{H_{j}} 2^{-n H_{j}} \quad \text { and } \quad B=c_{2,1}^{1 / 2}(k+2)^{H_{j}} 2^{-n H_{j}} .
$$

Then $A$ is the infimum of $c_{2,1}^{1 / 2}\left|t_{j}-s_{j}\right|^{H_{j}}$ taken over $s_{j}, t_{j}$ who satisfy (4.8), and $B$ is the corresponding supremum. The parameters $k$ and $l$ will be restricted to the following ranges:

$$
a 2^{n}+1 \leq l \leq 2^{n}, \quad \frac{1}{4} n \leq k \leq n
$$

for $n=3,4, \ldots$. It is easy to check that

$$
0 \leq 1-A B^{-1} \leq c n^{-1} \rightarrow 0 .
$$

By taking $x_{j}=\frac{l-1}{2^{n}}, T_{j}=a_{j}=\frac{k+2}{2^{n}}$ and $u=\frac{(1+\mu) c_{4,2} c_{2,1}^{1 / 2}}{N} \sqrt{\log \left(1+B^{-1}\right)}$ in (3.7), we obtain that for $n$ large enough

$$
\begin{aligned}
\mathbb{P}\left(E_{j}(l, k, n)\right) & \leq c\left(\frac{2^{n}}{k+2}\right)^{\frac{(N-1) H_{j}}{\min \left\{H_{i}\right\}}} \exp \left(-\frac{(1+\mu / 2)^{2} c_{4,2}^{2} c_{2,1} \log \left(1+B^{-1}\right)}{2 N^{2} c_{2,2}}\right) \\
& \leq c\left(\frac{n}{2^{n}}\right)^{\frac{(1+\mu / 2)^{2} c_{4,2}^{2} c_{2,1} H_{j}}{2 N^{2} c_{2,2}}}-\frac{(N-1) H_{j}}{\min \left\{H_{i}\right\}} .
\end{aligned}
$$


Thus

$$
\sum_{n=1}^{\infty} \sum_{l, k} \mathbb{P}\left(E_{j}(l, k, n)\right) \leq c \sum_{n=1}^{\infty} n 2^{n}\left(\frac{n}{2^{n}}\right)^{\frac{(1+\mu / 2)^{2} c_{4,2}^{2} c_{2,1} H_{j}}{2 N^{2} c_{2,2}}-\frac{(N-1) H_{j}}{\min \left\{H_{i}\right\}}}<\infty,
$$

where the summation $\sum_{l, k}$ is taken over integers $l, k$ that satisfy (4.9) and, for obtaining the last inequality, we have used the definition of $c_{4,2}$. Thus, by the Borel-Cantelli lemma, there exists a.s. an integer $n_{0}=n_{0}(\omega)$ such that none of the events $E_{j}(l, k, n)$ occur for $n \geq n_{0}$ and $l, k$ satisfying (4.9).

Let $\left[s_{j}, t_{j}\right]$ be an interval with $t_{j}-s_{j} \leq n_{0} 2^{-n_{0}}$. Now we first choose $n \geq n_{0}$ so that

$$
(n+1) 2^{-n-1}<t_{j}-s_{j} \leq n 2^{-n},
$$

and then $l$ and $k$ so that

$$
(l-1) 2^{-n} \leq s_{j}<l 2^{-n}, \quad(l+k) 2^{-n} \leq t_{j}<(l+k+1) 2^{-n} .
$$

It is now easy to check that if $\left[s_{j}, t_{j}\right] \subseteq[a, 1]$, then the indices $l, k$ satisfy (4.9) and $\left[s_{j}, t_{j}\right]$ is one of the intervals in the event $E_{j}(l, k, n)$. Hence $\lim \sup _{\varepsilon \rightarrow 0+} J_{j}(\varepsilon) \leq(1+\mu) c_{4,2} / N$ a.s. Letting $\mu \downarrow 0$, yields (4.7) for every $1 \leq j \leq N$. Combining (4.6) and (4.7), we obtain (4.4) immediately.

Now we show (4.5). Let $1<\theta<2$ be a constant which will be specified later and, for all $n \geq 1$, let

$$
\varepsilon_{n}=\sqrt{c_{2,2} \sum_{j=1}^{N} \theta^{-2 H_{j} n}}
$$

Since for any $0<\varepsilon<1$ there is an integer $n \geq 2$ such that $\varepsilon_{n}<\varepsilon \leq \varepsilon_{n-1}$, by the monotonicity of $J(\varepsilon)$ and condition (A1), we have

$$
\begin{aligned}
\lim _{\varepsilon \rightarrow 0+} J(\varepsilon) & =\lim _{n \rightarrow \infty} \sup _{s, t \in I, \sigma(s, t) \leq \varepsilon_{n}} \frac{|X(t)-X(s)|}{\beta(s, t)} \\
& \geq \liminf _{n \rightarrow \infty} \max _{a \theta^{n} / 2 \leq i \leq\left(\theta^{n}-1\right) / 2} \frac{\left|X\left(\left\langle(2 i+1) \theta^{-n}\right\rangle\right)-X\left(\left\langle 2 i \theta^{-n}\right\rangle\right)\right|}{\varepsilon_{n} \sqrt{\log \left(1+\varepsilon_{n}^{-1}\right)}} \\
& =: \liminf _{n \rightarrow \infty} J_{n} .
\end{aligned}
$$

Recall that $\langle c(i)\rangle$ means the $N$-dimensional vector $(c(i), \ldots, c(i))$.

For any $\mu \in(0,1)$, we have

$$
\begin{aligned}
& \mathbb{P}\left(J_{n} \leq(1-\mu) c_{4,1}\right) \\
& \leq \mathbb{P}\left(\left\{\frac{\left|X(\langle 1\rangle)-X\left(\left\langle 1-\theta^{-n}\right\rangle\right)\right|}{\left.\varepsilon_{n} \sqrt{\log \left(1+\varepsilon_{n}^{-1}\right)} \leq(1-\mu) c_{4,1}\right\}}\right.\right. \\
& \qquad \max _{\substack{a \theta^{n} / 2 \leq i \leq\left(\theta^{n}-1\right) / 2-1 \\
\varepsilon_{n} \sqrt{\log \left(1+\varepsilon_{n}^{-1}\right)}}} \frac{\left|X\left(\left\langle(2 i+1) \theta^{-n}\right\rangle\right)-X\left(\left\langle 2 i \theta^{-n}\right\rangle\right)\right|}{P_{1}(n) \cdot P_{2}(n),}
\end{aligned}
$$

where

$$
P_{1}(n)=\mathbb{P}\left(\max _{a \theta^{n} / 2 \leq i \leq\left(\theta^{n}-1\right) / 2-1} \frac{\left|X\left(\left\langle(2 i+1) \theta^{-n}\right\rangle\right)-X\left(\left\langle 2 i \theta^{-n}\right\rangle\right)\right|}{\varepsilon_{n} \sqrt{\log \left(1+\varepsilon_{n}^{-1}\right)}} \leq(1-\mu) c_{4,1}\right)
$$


and

$$
\begin{array}{r}
P_{2}(n)=\mathbb{P}\left(\frac{\left|X(\langle 1\rangle)-X\left(\left\langle 1-\theta^{-n}\right\rangle\right)\right|}{\varepsilon_{n} \sqrt{\log \left(1+\varepsilon_{n}^{-1}\right)} \leq}(1-\mu) c_{4,1} \mid X\left(\left\langle 1-\theta^{-n}\right\rangle\right) ; X\left(\left\langle(2 i+1) \theta^{-n}\right\rangle\right),\right. \\
\left.X\left(\left\langle 2 i \theta^{-n}\right\rangle\right), a \theta^{n} / 2 \leq i \leq\left(\theta^{n}-1\right) / 2-1\right) .
\end{array}
$$

By condition (A2), we have

$$
\begin{aligned}
& \operatorname{Var}\left(X(\langle 1\rangle)-X\left(\left\langle 1-\theta^{-n}\right\rangle\right) \mid X\left(\left\langle 1-\theta^{-n}\right\rangle\right) ; X\left(\left\langle(2 i+1) \theta^{-n}\right\rangle\right),\right. \\
& \left.X\left(\left\langle 2 i \theta^{-n}\right\rangle\right), a \theta^{n} / 2 \leq i \leq\left(\theta^{n}-1\right) / 2-1\right) \\
& \geq c_{2,3} \sum_{j=1}^{N} \theta^{-2 H_{j} n}=\frac{c_{2,3}}{c_{2,2}} \varepsilon_{n}^{2} .
\end{aligned}
$$

Thus, by the fact that the conditional distributions of the Gaussian process are still Gaussian and by Anderson's inequality (see Anderson [1]), we derive

$$
P_{2}(n) \leq \mathbb{P}\left(N(0,1) \leq(1-\mu) c_{4,1} \sqrt{\left(c_{2,2} / c_{2,3}\right) \log \left(1+\varepsilon_{n}^{-1}\right)}\right),
$$

where $N(0,1)$ denotes a standard normal random variable. By using the following well-known inequality

$$
(2 \pi)^{-\frac{1}{2}}\left(1-x^{-2}\right) x^{-1} e^{-\frac{x^{2}}{2}} \leq \mathbb{P}(N(0,1)>x) \leq(2 \pi)^{-\frac{1}{2}} x^{-1} e^{-\frac{x^{2}}{2}}, \quad \forall x>0,
$$

we derive that for all $n$ large enough

$$
\begin{aligned}
P_{2}(n) & =1-\mathbb{P}\left(N(0,1)>(1-\mu) c_{4,1} \sqrt{\left(c_{2,2} / c_{2,3}\right) \log \left(1+\varepsilon_{n}^{-1}\right)}\right) \\
& \leq 1-\varepsilon_{n} \frac{(1-\mu / 2)^{2} c_{2,2} 2 c_{4,1}^{2}}{2 c_{2,3}} \\
& \leq \exp \left(-\frac{(1-\mu / 2)^{2} c_{2,2} c_{4,1}^{2}}{2 c_{2,3}}\right),
\end{aligned}
$$

where for obtaining the last inequality we have used the elementary inequality: $\forall x$, $1-x \leq e^{-x}$. Hence

$$
\mathbb{P}\left(J_{n} \leq(1-\mu) c_{4,1}\right) \leq \exp \left(-\varepsilon_{n}^{\frac{(1-\mu / 2)^{2} c_{2,2} c_{4,1}^{2}}{2 c_{2,3}}}\right) \cdot P_{1}(n) .
$$

By repeating the above argument, we derive that

$$
\begin{aligned}
\mathbb{P}\left(J_{n} \leq(1-\mu) c_{4,1}\right) & \leq \exp \left(-\frac{(1-a) \theta^{n}-1}{2} \frac{\frac{(1-\mu / 2)^{2} c_{2,2} c_{4,1}^{2}}{2 c_{2,3}}}{\varepsilon_{n}}\right) \\
& \leq \exp \left(-c \theta^{n} \theta^{-\frac{\min \left\{H_{i}\right\}(1-\mu / 2)^{2} c_{2,2} c_{4,1}^{2} n}{2 c_{2,3}}}\right),
\end{aligned}
$$

where the last inequality follows from the estimate: $\varepsilon_{n}^{2} \geq c_{2,2} \theta^{-2 \min \left\{H_{i}\right\} n}$. Thus, by the definition of $c_{4,1}$, we get

$$
\sum_{n=1}^{\infty} \mathbb{P}\left(J_{n} \leq(1-\mu) c_{4,1}\right)<\infty .
$$


Thus, the Borel-Cantelli lemma implies

$$
\liminf _{n \rightarrow \infty} J_{n} \geq(1-\mu) c_{4,1} \quad \text { a.s. }
$$

Letting $\mu \downarrow 0$ along a sequence, by (4.10) and (4.12) we obtain (4.5). The proof of Theorem 4.1 is completed.

\section{LOCAL MODULI OF CONTINUITY, LAWS OF THE ITERATED LOGARITHM}

In this section we investigate the local moduli of continuity for anisotropic random fields with stationary increments. Let $X=\left\{X(t), t \in \mathbb{R}^{N}\right\}$ be a real-valued centered Gaussian random field with $X(\langle 0\rangle)=0$. We assume that the covariance function $R(s, t)=\mathbb{E}[X(s) X(t)]$ is continuous and $X$ has stationary increments. The latter means that for any $h \in \mathbb{R}^{N}$,

$$
\left\{X(t+h)-X(h), t \in \mathbb{R}^{N}\right\} \stackrel{d}{=}\left\{X(t), t \in \mathbb{R}^{N}\right\},
$$

where $\stackrel{d}{=}$ means equality in finite-dimensional distributions. According to Yaglom [33], $R(s, t)$ can be represented as

$$
R(s, t)=\int_{\mathbb{R}^{N}}\left(e^{i\langle s, \xi\rangle}-1\right)\left(e^{i\langle t, \xi\rangle}-1\right) \Delta(d \xi)+\langle s, Q t\rangle,
$$

where $Q=\left(q_{i j}\right)$ is an $N \times N$ non-negative definite matrix and $\Delta(d \xi)$ is a nonnegative symmetric measure on $\mathbb{R}^{N} \backslash\{0\}$ satisfying

$$
\int_{\mathbb{R}^{N}} \frac{|\xi|^{2}}{1+|\xi|^{2}} \Delta(d \xi)<\infty
$$

The measure $\Delta$ and its density $f(\xi)$ (if it exists) are called the spectral measure and spectral density of $X$, respectively.

It follows from (5.1) that $X$ has the stochastic integral representation

$$
X(t)=\int_{\mathbb{R}^{N}}\left(e^{i\langle t, \xi\rangle}-1\right) \mathcal{M}(d \xi)+\langle\mathbf{Y}, t\rangle,
$$

where $\mathbf{Y}$ is an $N$-dimensional Gaussian random vector with mean zero and $\mathcal{M}(d \xi)$ is a centered complex-valued Gaussian random measure which is independent of $\mathbf{Y}$ and satisfies

$$
\mathbb{E}[\mathcal{M}(A) \overline{\mathcal{M}(B)}]=\Delta(A \cap B) \quad \text { and } \quad \mathcal{M}(-A)=\overline{\mathcal{M}(A)}
$$

for all Borel sets $A, B \subset \mathbb{R}^{N}$ with finite $\Delta$-measure. The spectral measure $\Delta$ is called the control measure of $\mathcal{M}$. Since the linear term $\langle\mathbf{Y}, t\rangle$ in (5.3) will not have any effect on the problems considered in this paper, we will from now on assume $\mathbf{Y}=0$. This is equivalent to assuming $Q=0$ in (5.1). Consequently, we have

$$
\sigma^{2}(h)=\mathbb{E}\left[(X(t+h)-X(t))^{2}\right]=2 \int_{\mathbb{R}^{N}}(1-\cos \langle h, \xi\rangle) \Delta(d \xi) .
$$

For $t_{0} \in \mathbb{R}^{N}$ and a family of neighborhoods $\{O(\delta), \delta>0\}$ of $\langle 0\rangle \in \mathbb{R}^{N}$ whose diameters go to 0 as $\delta \rightarrow 0$, we consider the corresponding local moduli of continuity of $X$ at $t_{0}$

$$
\omega\left(t_{0}, \delta\right)=\sup _{s \in O(\delta)}\left|X\left(t_{0}+s\right)-X\left(t_{0}\right)\right| .
$$

It can be seen that if $X$ is anisotropic, then the rate at which $\omega\left(t_{0}, \delta\right)$ goes to 0 as $\delta \rightarrow 0$ depends upon the shape of $O(\delta)$. 
In the following, we consider two kinds of local moduli of continuity for $X$. Theorem 5.1 is concerned with the local modulus of continuity measured in the most general way. Theorem 5.6 provides the local modulus of continuity in the metric $\sigma$. It should be noted that the logarithmic factors in these two theorems are quite different, since (A1) implies $\sigma(s) \asymp \sum_{j=1}^{N}\left|s_{j}\right|^{H_{j}}$ as $s \rightarrow 0$ (ratio remains bounded away from zero and infinity) in Theorem 5.6, and the corresponding term $\prod_{j=1}^{N}\left|s_{j}\right|^{H_{j}}$ in Theorem 5.1 is much smaller.

Theorem 5.1. Let $\left\{X(t), t \in \mathbb{R}^{N}\right\}$ be a real-valued centered Gaussian random field with stationary increments and $X(\langle 0\rangle)=0$. If $X$ satisfies condition (A1) for $I=[0,1]^{N}$, then there is a positive constant $\kappa_{2}$ such that for every $t_{0} \in \mathbb{R}^{N}$ we have

$$
\limsup _{\|\varepsilon\| \rightarrow 0+\left\langle\left|s_{j}\right|\right\rangle \leq\left\langle\varepsilon_{j}\right\rangle} \frac{\left|X\left(t_{0}+s\right)-X\left(t_{0}\right)\right|}{\gamma(s)}=\kappa_{2} \quad \text { a.s. }
$$

where

$$
\gamma(s)=\sigma(s)\left[\log \log \left(1+\frac{1}{\prod_{j=1}^{N}\left|s_{j}\right|^{H_{j}}}\right)\right]^{\frac{1}{2}}, \quad \forall s \in \mathbb{R}^{N} .
$$

Remark 5.2. Equation (5.5) means that for any $\eta>0$, there exists a.s. $\delta_{0}=\delta_{0}(\omega)>$ 0 such that for all $\varepsilon \in(0,1)^{N}$ that satisfies $\|\varepsilon\| \leq \delta_{0}$, we have

$$
\sup _{\left\langle\left|s_{j}\right|\right\rangle \leq\left\langle\varepsilon_{j}\right\rangle} \frac{\left|X\left(t_{0}+s\right)-X\left(t_{0}\right)\right|}{\gamma(s)}<\kappa_{2}+\eta .
$$

Moreover, for any $\eta>0$ there exists a sequence $\varepsilon(n) \in(0,1)^{N}$ such that $\|\varepsilon(n)\| \rightarrow 0$ and

for $n$ large enough.

$$
\sup _{\left\langle\left|s_{j}\right|\right\rangle \leq \varepsilon(n)} \frac{\left|X\left(t_{0}+s\right)-X\left(t_{0}\right)\right|}{\gamma(s)}>\kappa_{2}-\eta
$$

In order to show Theorem 5.1, we will make use of the following lemmas. The first one (Lemma 5.3) is taken from Talagrand [28. Let $\{Z(t), t \in S\}$ be a centered Gaussian process with values in $\mathbb{R}$. The index set $S$ is equipped with the pseudometric $d(s, t)=\left[\mathbb{E}(Z(t)-Z(s))^{2}\right]^{1 / 2}$. We denote by $N_{d}(S, \varepsilon)$ the smallest number of (open) $d$-balls of radius $\varepsilon$ needed to cover $S$ and we denote by $D$ the diameter of $S$, that is, $D=\sup \{d(s, t): s, t \in S\}$.

Lemma 5.3. Given $x>0$, we have

$$
\mathbb{P}\left(\sup _{s, t \in S}|Z(t)-Z(s)| \geq c_{5,1}\left(x+\int_{0}^{D} \sqrt{\log N_{d}(S, \varepsilon)} d \varepsilon\right)\right) \leq \exp \left(-\frac{x^{2}}{D^{2}}\right)
$$

where $c_{5,1}$ is a positive and finite constant.

Lemma 5.4. Let $\left\{X(t), t \in \mathbb{R}^{N}\right\}$ be a real-valued centered Gaussian random field satisfying the upper bound in condition (A1). Then there exist positive and finite constants $u_{0}$ and $c_{5,2}$ such that for all $t_{0} \in I$ and $u \geq u_{0}$,

$$
\mathbb{P}\left(\sup _{\left\langle\left|s_{j}\right|\right\rangle \leq\left\langle a_{j}\right\rangle}\left|X\left(t_{0}+s\right)-X\left(t_{0}\right)\right| \geq u \sum_{j=1}^{N} a_{j}^{H_{j}}\right) \leq e^{-c_{5,2} u^{2}}
$$

for all $\left\langle a_{j}\right\rangle \in(0,1]^{N}$ such that $t_{0}-\left\langle a_{j}\right\rangle \in I$ and $t_{0}+\left\langle a_{j}\right\rangle \in I$. 
Proof. We will use Lemma 5.3 to prove Lemma 5.4. Consider the Gaussian random field $\{Z(t), t \in S\}$ defined by $Z(t)=X\left(t_{0}+t\right)-X\left(t_{0}\right)$, where $S=\left\{t \in \mathbb{R}^{N}:\left|t_{j}\right| \leq\right.$ $\left.a_{j}\right\}$. By condition (A1), we have

$$
d(s, t)=d_{Z}(s, t) \leq c_{2,2} \sum_{j=1}^{N}\left|s_{j}-t_{j}\right|^{H_{j}}, \quad \forall s, t \in S .
$$

Thus

$$
N_{d}(S, \varepsilon) \leq c\left(\prod_{j=1}^{N} a_{j}\right) \varepsilon^{-\sum_{j=1}^{N} \frac{1}{H_{j}}},
$$

and the diameter $D$ of $S$ is at most $c \sum_{j=1}^{N} a_{j}^{H_{j}}$. Let $j_{0}$ be the index such that $a_{j_{0}}^{H_{j_{0}}}=\max \left\{a_{j}^{H_{j}}, 1 \leq j \leq N\right\}$. It is elementary to verify that

$$
\begin{aligned}
\int_{0}^{D} \sqrt{\log N_{d}(S, \varepsilon)} d \varepsilon & \leq c \int_{0}^{D} \sqrt{\log \left(\prod_{j=1}^{N} \frac{a_{j}}{\varepsilon^{1 / H_{j}}}\right)} d \varepsilon \\
& \leq c \int_{0}^{c N a_{j_{0}}^{H_{j}}} \sqrt{\log \left(\frac{a_{j_{0}}^{H_{j}}}{\varepsilon}\right)^{\sum_{j=1}^{N} \frac{1}{H_{j}}}} d \varepsilon \\
& =c a_{j_{0}}^{H_{j_{0}}} \int_{0}^{c N} \sqrt{\log \left(\frac{1}{\eta}\right)} d \eta \\
& \leq c \sum_{j=1}^{N} a_{j}^{H_{j}} .
\end{aligned}
$$

Hence the conclusion of the lemma follows from (5.7).

The following truncation inequalities are extensions of those in Loéve [18, p. 209] for $N=1$ and (3.4) and (3.5) in Xiao 31 for $N>1$ and $\rho$ being replaced by the Euclidean metric. In the current form, they are proved in Luan and Xiao [19].

Lemma 5.5. Let $\Delta$ be a non-negative symmetric Borel measure on $\mathbb{R}^{N} \backslash\{0\}$ which satisfies (5.2). Then for any $u>0$ and any $t \in \mathbb{R}^{N}$ with $\rho(0, t) u \leq 1 / N$ we have

$$
\int_{\{\xi: \rho(0, \xi)<u\}}\langle t, \xi\rangle^{2} \Delta(d \xi) \leq c \int_{\mathbb{R}^{N}}(1-\cos \langle t, \xi\rangle) \Delta(d \xi),
$$

and for all $u>0$

$$
\int_{\{\xi: \rho(0, \xi) \geq u\}} \Delta(d \xi) \leq c u^{Q} \int_{\{v: \rho(0, v) \leq 1 / u\}} d v \int_{\mathbb{R}^{N}}(1-\cos \langle v, \xi\rangle) \Delta(d \xi),
$$

where $Q=\sum_{j=1}^{N} \frac{1}{H_{j}}$.

We are in position to prove Theorem 5.1. Due to the stationarity of increments of $X$, it is sufficient to consider $t_{0}=\langle 0\rangle$. However, we will keep writing $t_{0}$ because the method for proving Theorem 5.1 remains valid as long as $X$ has an appropriate stochastic integral representation. In particular, we will see in Section 6 that the proof below can be modified to obtain local moduli of continuity for fractional Brownian sheets, which do not have stationary increments in the usual sense. 
Proof of Theorem 5.1. For any $\varepsilon=\left\langle\varepsilon_{j}\right\rangle \in(0,1)^{N}$, put

$$
M(\varepsilon)=\sup _{\left\langle\left|s_{j}\right|\right\rangle \leq\left\langle\varepsilon_{j}\right\rangle} \frac{\left|X\left(t_{0}+s\right)-X\left(t_{0}\right)\right|}{\gamma(s)} .
$$

Note that, even though $M(\varepsilon)$ is a non-decreasing function of $\varepsilon \in(0,1)^{N}$ in the partial order $\leq$, it is in general not monotone in $\|\varepsilon\|$. We claim that

$$
\limsup _{\|\varepsilon\| \rightarrow 0+} M(\varepsilon) \leq c_{5,3} \quad \text { a.s. }
$$

for some constant $c_{5,3}>0$ and

$$
\limsup _{\|\varepsilon\| \rightarrow 0+} M(\varepsilon) \geq \sqrt{2} \quad \text { a.s. }
$$

Before proving (5.11) and (5.12), let us note again that (5.11) and the proof of Lemma 7.1.1 in Marcus and Rosen [20] imply (5.5) and the constant $\kappa_{2} \in\left[\sqrt{2}, c_{5,3}\right]$.

Hence, it is enough to verify (5.11) and (5.12). We show (5.11) first. For any $\mathbf{n}=\left(n_{1}, \ldots, n_{N}\right) \in \mathbb{N}^{N}$, let $h_{\mathbf{n}}=\left\langle 2^{-n_{j}}\right\rangle$. Let $\delta>0$ be a constant whose value will be determined later. Define the event

$$
F_{\mathbf{n}}=\left\{\sup _{h_{\mathbf{n}} \leq\left\langle\left|s_{j}\right|\right\rangle \leq h_{\mathbf{n}-\langle 1\rangle}} \gamma(s)^{-1}\left|X\left(t_{0}+s\right)-X\left(t_{0}\right)\right| \geq \delta\right\} .
$$

By condition (A1), we see that for any $s \in \mathbb{R}^{N}$ that satisfies $h_{\mathbf{n}} \leq\left\langle\left|s_{j}\right|\right\rangle \leq h_{\mathbf{n}-\langle 1\rangle}$, we have

$$
\gamma(s) \geq c_{2,1}\left(\sum_{j=1}^{N} 2^{-n_{j} H_{j}}\right) \sqrt{\log \log \left(1+\prod_{j=1}^{N} 2^{\left(n_{j}-1\right) H_{j}}\right)} .
$$

This and Lemma 5.4 imply

$$
\begin{aligned}
\mathbb{P}\left(F_{\mathbf{n}}\right) & \leq \exp \left(-c_{5,4} \delta^{2} \log \log \left(1+\prod_{j=1}^{N} 2^{\left(n_{j}-1\right) H_{j}}\right)\right) \\
& \leq c\left(\sum_{j=1}^{N} n_{j}\right)^{-c_{5,4} \delta^{2}},
\end{aligned}
$$

where $c_{5,4}=c_{5,2} c_{2,1}^{2}$. By taking $\delta$ large enough such that $c_{5,4} \delta^{2}>N$, we see that

$$
\sum_{\mathbf{n} \in \mathbb{N}^{N}} \mathbb{P}\left(F_{\mathbf{n}}\right) \leq c \sum_{\mathbf{n} \in \mathbb{N}^{N}}\|\mathbf{n}\|^{-c_{5,4} \delta^{2}}<\infty
$$

Thus, by the Borel-Cantelli lemma, a.s. only finitely many of the events $F_{\mathbf{n}}$ occur. This implies

$$
\limsup _{\|\mathbf{n}\| \rightarrow \infty} \sup _{h_{\mathbf{n}} \leq\left\langle\left|s_{j}\right|\right\rangle \leq h_{\mathbf{n}-\langle 1\rangle}} \frac{\left|X\left(t_{0}+s\right)-X\left(t_{0}\right)\right|}{\gamma(s)} \leq \delta \quad \text { a.s. }
$$

Let $s \in \mathbb{R}^{N}$ be a point such that for some $\mathbf{n}^{0} \in \mathbb{N}^{N}$ we have $\left|s_{j}\right| \leq 2^{-n_{j}^{0}}$ for every $1 \leq j \leq N$. Now we choose $\mathbf{n} \in \mathbb{N}^{N}$ so that

$$
2^{-n_{j}}<\left|s_{j}\right| \leq 2^{-n_{j}+1}
$$

for every $1 \leq j \leq N$. This and (5.13) yield (5.11). 
Now we show that (5.12) holds. For this purpose, it is sufficient to provide a sequence $\left\langle\varepsilon_{j}^{(n)}\right\rangle \in(0,1)^{N}$ such that $\left\|\left\langle\varepsilon_{j}^{(n)}\right\rangle\right\| \rightarrow 0$ and

$$
\limsup _{n \rightarrow \infty} \frac{\left|X\left(t_{0}+\left\langle\varepsilon_{j}^{(n)}\right\rangle\right)-X\left(t_{0}\right)\right|}{\gamma\left(\left\langle\varepsilon_{j}^{(n)}\right\rangle\right)} \geq \sqrt{2} \quad \text { a.s. }
$$

To this end we will use the spectral representation (5.3) of $X$ to create independence among the random variables. This argument is a modification of those in Monrad and Rootzén [21, Talagrand [28, or Li and Shao [17] so that it adapts to the anisotropy of $X$.

For any $0<\mu<1$ and $n \geq 1$, we define $\left\langle\varepsilon_{j}^{(n)}\right\rangle=\left(\varepsilon_{1}^{(n)}, \ldots, \varepsilon_{N}^{(n)}\right)$ by

$$
\varepsilon_{j}^{(n)}=\exp \left(-H_{j}^{-1} n^{1+\mu}\right) \quad(j=1, \ldots, N) .
$$

Then $\rho\left(0,\left\langle\varepsilon_{j}^{(n)}\right\rangle\right)=N \exp \left(-n^{1+\mu}\right)$.

For every integer $n \geq 1$, we denote

$$
d_{n}=\exp \left(n^{1+\mu}+n^{\mu}\right)
$$

and define the Gaussian random fields

$$
\widetilde{X}_{n}(t)=\int_{\left\{\rho(0, \xi) \notin\left(d_{n-1}, d_{n}\right]\right\}}\left(e^{i\langle t, \xi\rangle}-1\right) \mathcal{M}(d \xi), \quad \forall t \in \mathbb{R}^{N}
$$

and

$$
X_{n}(t)=\int_{\left\{\rho(0, \xi) \in\left(d_{n-1}, d_{n}\right]\right\}}\left(e^{i\langle t, \xi\rangle}-1\right) \mathcal{M}(d \xi), \quad \forall t \in \mathbb{R}^{N} .
$$

Then $\left\{\widetilde{X}_{n}(t), t \in \mathbb{R}^{N}\right\}$ and $\left\{X_{n}(t), t \in \mathbb{R}^{N}\right\}$ are independent and $X(t)=X_{n}(t)+$ $\widetilde{X}_{n}(t)$ for all $t \in \mathbb{R}^{N}$. Moreover, the random fields $\left\{X_{n}(t), t \in \mathbb{R}^{N}\right\}, n=1,2, \ldots$, are independent. Note that

$$
\begin{aligned}
& \limsup _{n \rightarrow \infty} \frac{\left|X\left(t_{0}+\left\langle\varepsilon_{j}^{(n)}\right\rangle\right)-X\left(t_{0}\right)\right|}{\gamma\left(\left\langle\varepsilon_{j}^{(n)}\right\rangle\right)} \\
& \geq \limsup _{n \rightarrow \infty} \frac{\left|X_{n}\left(t_{0}+\left\langle\varepsilon_{j}^{(n)}\right\rangle\right)-X_{n}\left(t_{0}\right)\right|}{\gamma\left(\left\langle\varepsilon_{j}^{(n)}\right\rangle\right)}-\limsup _{n \rightarrow \infty} \frac{\left|\widetilde{X}_{n}\left(t_{0}+\left\langle\varepsilon_{j}^{(n)}\right\rangle\right)-\widetilde{X}_{n}\left(t_{0}\right)\right|}{\gamma\left(\left\langle\varepsilon_{j}^{(n)}\right\rangle\right)} \\
& =: \limsup _{n \rightarrow \infty} I_{1}(n)-\limsup _{n \rightarrow \infty} I_{2}(n) .
\end{aligned}
$$

By the definition of $\widetilde{X}_{n}(t)$ we have

$$
\begin{aligned}
& \mathbb{E}\left(\widetilde{X}_{n}\left(t_{0}+\left\langle\varepsilon_{j}^{(n)}\right\rangle\right)-\widetilde{X}_{n}\left(t_{0}\right)\right)^{2} \\
& =2\left(\int_{\rho(0, \xi) \leq d_{n-1}}+\int_{\rho(0, \xi)>d_{n}}\right)\left(1-\cos \left\langle\left\langle\varepsilon_{j}^{(n)}\right\rangle, \xi\right\rangle\right) \Delta(d \xi) \\
& \leq \int_{\rho(0, \xi) \leq d_{n-1}}\left\langle\varepsilon_{j}^{(n)}, \xi\right\rangle^{2} \Delta(d \xi)+4 \int_{\rho(0, \xi)>d_{n}} \Delta(d \xi) .
\end{aligned}
$$

To derive the above inequality, we bound $1-\cos \langle t, x\rangle$ by $\langle t, x\rangle^{2} / 2$ and by 2 , respectively. 
Now we estimate the last two integrals separately. Denote $U=\exp \left(\mu(n-1)^{\mu}\right)$. Note that

$$
\begin{aligned}
\rho\left(0,\left\langle\varepsilon_{j}^{(n)} U\right\rangle\right) d_{n-1} & \leq N U^{\max \left\{H_{i}\right\}} \exp \left(-n^{1+\mu}+(n-1)^{1+\mu}+(n-1)^{\mu}\right) \\
& \leq N \exp \left(-\mu\left(1-\max \left\{H_{i}\right\}\right)(n-1)^{\mu}\right),
\end{aligned}
$$

which is smaller than $1 / N$ for $n$ large. It follows from (5.9) that

$$
\begin{aligned}
\int_{\rho(0, \xi) \leq d_{n-1}}\left\langle\left\langle\varepsilon_{j}^{(n)}\right\rangle, \xi\right\rangle^{2} \Delta(d \xi) & =U^{-2} \int_{\rho(0, \xi) \leq d_{n-1}}\left\langle\left\langle\varepsilon_{j}^{(n)} U\right\rangle, \xi\right\rangle^{2} \Delta(d \xi) \\
& \leq c U^{-2} \sigma^{2}\left(\left\langle\varepsilon_{j}^{(n)} U\right\rangle\right) \\
& \leq c U^{-2\left(1-\max \left\{H_{i}\right\}\right)} \rho\left(0,\left\langle\varepsilon_{j}^{(n)}\right\rangle\right)^{2},
\end{aligned}
$$

where the last inequality follows from condition (A1).

On the other hand, (5.10) and condition (A1) imply that

$$
\begin{aligned}
\int_{\rho(0, \xi)>d_{n}} \Delta(d \xi) & \leq c d_{n}^{Q} \int_{v: \rho(0, v) \leq d_{n}^{-1}} \sigma^{2}(v) d v \\
& \leq c d_{n}^{-2}=c \rho\left(0,\left\langle\varepsilon_{j}^{(n)}\right\rangle\right)^{2} \exp \left(-2 n^{\mu}\right) .
\end{aligned}
$$

Combining (5.18) and (5.19), we obtain

$$
\begin{aligned}
\mathbb{E}\left(\widetilde{X}_{n}\left(t_{0}+\left\langle\varepsilon_{j}^{(n)}\right\rangle\right)-\widetilde{X}_{n}\left(t_{0}\right)\right)^{2} & \leq c\left(U^{-2\left(1-\max \left\{H_{i}\right\}\right)} \rho\left(0,\left\langle\varepsilon_{j}^{(n)}\right\rangle\right)^{2}+d_{n}^{-2}\right) \\
& \leq c \rho\left(0,\left\langle\varepsilon_{j}^{(n)}\right\rangle\right)^{2} \exp \left(-2\left(1-\max \left\{H_{i}\right\}\right) \mu n^{\mu}\right) .
\end{aligned}
$$

Hence for any $\eta>0$, we have

$$
\begin{aligned}
\mathbb{P}\left(I_{2}(n) \geq \eta\right) & \leq \mathbb{P}\left(\left|\widetilde{X}_{n}\left(t_{0}+\left\langle\varepsilon_{j}^{(n)}\right\rangle\right)-\widetilde{X}_{n}\left(t_{0}\right)\right| \geq \eta \gamma\left(\left\langle\varepsilon_{j}^{(n)}\right\rangle\right)\right) \\
& \leq \mathbb{P}\left(|N(0,1)| \geq \eta \sqrt{\log n} \exp \left(\left(1-\max \left\{H_{i}\right\}\right) \mu n^{\mu}\right)\right) \\
& \leq n^{-2}
\end{aligned}
$$

for all $n$ large enough. Thus $\sum_{n=1}^{\infty} \mathbb{P}\left(I_{2}(n) \geq \eta\right)<\infty$. By the Borel-Cantelli lemma and the arbitrariness of $\eta$, we obtain

$$
\limsup _{n \rightarrow \infty} I_{2}(n)=0 \quad \text { a.s. }
$$

In order to estimate $\limsup _{n \rightarrow \infty} I_{1}(n)$, note that

$$
\mathbb{E}\left(X_{n}\left(t_{0}+\left\langle\varepsilon_{j}^{(n)}\right\rangle\right)-X_{n}\left(t_{0}\right)\right)^{2} \leq \sigma^{2}\left(0,\left\langle\varepsilon_{j}^{(n)}\right\rangle\right) .
$$

It follows from this and (4.11) that for any $0<\eta<1$,

$$
\begin{aligned}
& \mathbb{P}\left(\left|X_{n}\left(t_{0}+\left\langle\varepsilon_{j}^{(n)}\right\rangle\right)-X_{n}\left(t_{0}\right)\right| \geq(1-\eta) \sqrt{2} \gamma\left(\left\langle\varepsilon_{j}^{(n)}\right\rangle\right)\right) \\
& \geq \mathbb{P}\left(|N(0,1)| \geq(1-\eta) \sqrt{2 \log \log \left(1+\exp \left(N n^{1+\mu}\right)\right.}\right) \\
& \geq c n^{-(1-\eta)^{2}(1+\mu)} .
\end{aligned}
$$

Now we choose $\mu>0$ small such that $(1-\eta)^{2}(1+\mu)<1$ and consequently $\sum_{n=1}^{\infty} \mathbb{P}\left(I_{1}(n) \geq(1-\eta) \sqrt{2}\right)=\infty$. Since the events $\left\{I_{1}(n) \geq(1-\eta) \sqrt{2}\right\}(n=$ $1,2, \ldots)$ are independent, the Borel-Cantelli lemma and the arbitrariness of $\eta$ yield

$$
\limsup _{n \rightarrow \infty} I_{1}(n) \geq \sqrt{2} \quad \text { a.s. }
$$

Hence (5.12) follows from (5.17), (5.21), and (5.22). The proof of Theorem 5.1 is now completed. 
Combining Theorem 5.1 and Lemma 7.1.1 in Marcus and Rosen 20] we derive the following local modulus of continuity.

Theorem 5.6. Let $X=\left\{X(t), t \in \mathbb{R}^{N}\right\}$ be a real-valued centered Gaussian random field with stationary increments and $X(\langle 0\rangle)=0$. If $X$ satisfies condition (A1) for $I=[0,1]^{N}$, then there is a positive and finite constant $\kappa_{3}$ such that for every $t_{0} \in \mathbb{R}^{N}$ we have

$$
\lim _{\varepsilon \rightarrow 0+} \sup _{s: \sigma(s) \leq \varepsilon} \frac{\left|X\left(t_{0}+s\right)-X\left(t_{0}\right)\right|}{\sigma(s) \sqrt{\log \log \left(1+\sigma(s)^{-1}\right)}}=\kappa_{3} \quad \text { a.s. }
$$

Proof. It suffices to prove that there exists a finite constant $c$ such that

$$
\lim _{\varepsilon \rightarrow 0+} \sup _{s: \sigma(s) \leq \varepsilon} \frac{\left|X\left(t_{0}+s\right)-X\left(t_{0}\right)\right|}{\sigma(s) \sqrt{\log \log \left(1+\sigma(s)^{-1}\right)}} \leq c \quad \text { a.s. }
$$

and

$$
\lim _{\varepsilon \rightarrow 0+} \sup _{s: \sigma(s) \leq \varepsilon} \frac{\left|X\left(t_{0}+s\right)-X\left(t_{0}\right)\right|}{\sigma(s) \sqrt{\log \log \left(1+\sigma(s)^{-1}\right)}} \geq \sqrt{2} .
$$

Equation (5.24) can be proved by using Lemma 5.4 and the Borel-Cantelli lemma, which is simpler than the proof of (5.11). We omit the details. have

On the other hand, we notice that for $\left\langle\varepsilon_{j}^{(n)}\right\rangle$ as in the proof of Theorem 5.1, we

$$
\sigma\left(\left\langle\varepsilon_{j}^{(n)}\right\rangle\right) \sqrt{\log \log \left(1+\sigma\left(\left\langle\varepsilon_{j}^{(n)}\right\rangle\right)^{-1}\right)} \sim \gamma\left(\left\langle\varepsilon^{(n)}\right\rangle\right)
$$

as $n \rightarrow \infty$. It follows from (5.14) that

$$
\lim _{n \rightarrow \infty} \frac{\left|X\left(t_{0}+\left\langle\varepsilon_{j}^{(n)}\right\rangle\right)-X\left(t_{0}\right)\right|}{\sigma\left(\left\langle\varepsilon_{j}^{(n)}\right\rangle\right) \sqrt{\log \log \left(1+\sigma\left(\left\langle\varepsilon_{j}^{(n)}\right\rangle\right)^{-1}\right)}} \geq \sqrt{2},
$$

which implies (5.25). The proof is finished.

\section{Applications}

In this section we discuss some applications of our results. In particular we are concerned with the applications to fractional Brownian sheets and to the solutions of stochastic partial differential equations.

6.1. Applications to fractional Brownian sheets. Fractional Brownian sheets were first introduced by Kamont [15] who also studied some of their regularly properties. As applications of our results, we establish the global and local moduli of continuity for fractional Brownian sheet for points. Our results extend the related results of Orey and Pruitt 25, Ayache and Xiao [2], and Wang 29]. For a given vector $H=\left(H_{1}, \ldots, H_{N}\right)\left(0<H_{j}<1\right.$ for $\left.j=1, \ldots, N\right)$, a one-dimensional fractional Brownian sheet $B^{H}=\left\{B^{H}(t), t \in \mathbb{R}^{N}\right\}$ with Hurst index $H$ is a real-valued centered Gaussian random field with covariance function given by

$$
\mathbb{E}\left[B^{H}(s) B^{H}(t)\right]=\prod_{j=1}^{N} \frac{1}{2}\left(\left|s_{j}\right|^{2 H_{j}}+\left|t_{j}\right|^{2 H_{j}}-\left|s_{j}-t_{j}\right|^{2 H_{j}}\right), \quad s, t \in \mathbb{R}^{N} .
$$

It follows from (6.1) that $B^{H}$ is an anisotropic Gaussian random field and $B^{H}=0$ a.s. for every $t \in \mathbb{R}^{N}$ with at least one zero coordinate. Ayache and Xiao [2] and $\mathrm{Wu}$ and Xiao [30] showed that for every $\varepsilon \in(0,1)$, fractional Brownian sheets 
satisfy conditions (A1) and (A2) for all $I \subset[\varepsilon, \infty)^{N}$. Therefore, by Theorem 4.1, we have the following global modulus of continuity for fractional Brownian sheets. The bound is sharp. Ayache and Xiao 2 established a sharp upper bound for the global modulus of continuity of fractional Brownian sheets by using the wavelet method. Theorem 6.1 below not only gives its sharp lower bound, but also improves the upper bound of Ayache and Xiao 2]. Wang [29] established a lower bound for the modulus of continuity of fractional Brownian sheets, but the bound is not as sharp as that given by Theorem 6.1. Theorem 6.2 is the local moduli of continuity or laws of the iterated logarithm for fractional Brownian sheets, which is complementary to Theorem 2 and Proposition 1 in Ayache and Xiao 2 .

Theorem 6.1. Let $\left\{B^{H}(t), t \in \mathbb{R}^{N}\right\}$ be a fractional Brownian sheet with index $H=\left(H_{1}, \ldots, H_{N}\right) \in(0,1)^{N}$, and let $I=[a, 1]^{N}$, where $a \in(0,1)$ is a constant. Then

$$
\lim _{\varepsilon \rightarrow 0+} \sup _{s, t \in I, \sigma(s, t) \leq \varepsilon} \frac{\left|B^{H}(t)-B^{H}(s)\right|}{\beta(s, t)}=\kappa_{4} \quad \text { a.s. },
$$

where $\beta(s, t)$ is defined as in (4.1) and $\kappa_{4}$ is a positive and finite constant.

Even though Theorems 5.1 and 5.6 cannot be applied directly to $B^{H}$ because it does not have stationary increments in the ordinary sense, one can apply the harmonizable representation of $B^{H}$ and modify the proof of Theorem 5.6 to prove Theorem 6.2

Theorem 6.2. Let $\left\{B^{H}(t), t \in \mathbb{R}^{N}\right\}$ be a fractional Brownian sheet with index $H=\left(H_{1}, \ldots, H_{N}\right) \in(0,1)^{N}$, and let $a \in(0,1)$ be a constant. Then for every $t_{0} \in[a, 1]^{N}$ there exist positive and finite constants $\kappa_{5}$ and $\kappa_{6}$ such that

$$
\limsup _{\|\varepsilon\| \rightarrow 0+\left\langle\left|s_{j}\right|\right\rangle \leq\left\langle\varepsilon_{j}\right\rangle} \sup _{\rho(0, s) \sqrt{\log \log \left(1+\prod_{j=1}^{N}\left|s_{j}\right|^{-H_{j}}\right)}}=\kappa_{5} \quad \text { a.s. }
$$

and

$$
\lim _{\varepsilon \rightarrow 0+} \sup _{s: \sigma(s) \leq \varepsilon} \frac{\left|B^{H}\left(t_{0}+s\right)-B^{H}\left(t_{0}\right)\right|}{\rho(0, s) \sqrt{\log \log \left(1+\rho(0, s)^{-1}\right)}}=\kappa_{6} \quad \text { a.s. }
$$

Remark 6.3. The constants $\kappa_{5}$ and $\kappa_{6}$ may depend on $t_{0}$, but they are bounded from above and below by positive constants which only depend on $H, a$, and $N$.

Proof of Theorem 6.2. The upper bounds in (6.3) and (6.4) follows respectively from the proofs of the upper bounds in Theorems 5.1 and 5.6, which only rely on condition (A1). For proving the lower bounds in (6.3) and (6.4), we need to modify the proofs of the lower bound in Theorem 5.1. Instead of using (5.3), we will make use of the harmonizable representation for $B^{H}$,

$$
B^{H}(t)=K_{H}^{-1} \int_{\mathbb{R}^{N}} \prod_{j=1}^{N} \frac{e^{i t_{j} \xi_{j}}-1}{\left|\xi_{j}\right|^{H_{j}+\frac{1}{2}}} \widetilde{W}(d \xi),
$$

where $K_{H}>0$ is a normalizing constant and $\widetilde{W}$ is a centered complex-valued Gaussian random measure in $\mathbb{R}^{N}$ with Lebesgue control measure. Or one may use the stochastic integral representation given by (2.6) in Wang [29]. 
Let $\left\langle\varepsilon_{j}^{(n)}\right\rangle$ and $d_{n}$ be defined as in the proof of Theorem 5.1. Similarly to (5.15) and (5.16), we define

$$
\widetilde{B}_{n}^{H}(t)=\int_{\rho(0, \xi) \notin\left(d_{n-1}, d_{n}\right]} \prod_{j=1}^{N} \frac{e^{i t_{j} \xi_{j}}-1}{\left|\xi_{j}\right|^{H_{j}+\frac{1}{2}}} \widetilde{W}(d \xi), \quad \forall t \in \mathbb{R}^{N},
$$

and

$$
B_{n}^{H}(t)=\int_{\rho(0, \xi) \in\left(d_{n-1}, d_{n}\right]} \prod_{j=1}^{N} \frac{e^{i t_{j} \xi_{j}}-1}{\left|\xi_{j}\right|^{H_{j}+\frac{1}{2}}} \widetilde{W}(d \xi), \quad \forall t \in \mathbb{R}^{N} .
$$

Then the random fields $\widetilde{B}_{n}^{H}$ and $B_{n}^{H}$ are independent. Moreover

$$
\begin{aligned}
& \mathbb{E}\left(\widetilde{B}_{n}^{H}\left(t_{0}+\left\langle\varepsilon_{j}^{(n)}\right\rangle\right)-\widetilde{B}_{n}^{H}\left(t_{0}\right)\right)^{2} \\
& \leq \int_{\rho(0, \xi) \leq d_{n-1}}\left|\prod_{j=1}^{N}\left(e^{i\left(t_{j}+\varepsilon_{j}^{(n)}\right) \xi_{j}}-1\right)-\prod_{j=1}^{N}\left(e^{i t_{j} \xi_{j}}-1\right)\right| \frac{d \xi}{\prod_{j=1}^{N}\left|\xi_{j}\right|^{2 H_{j}+1}} \\
& \quad \quad+\int_{\rho(0, \xi)>d_{n}}\left|\prod_{j=1}^{N}\left(e^{i\left(t_{j}+\varepsilon_{j}^{(n)}\right) \xi_{j}}-1\right)-\prod_{j=1}^{N}\left(e^{i t_{j} \xi_{j}}-1\right)\right| \frac{d \xi}{\prod_{j=1}^{N}\left|\xi_{j}\right|^{2 H_{j}+1}} \\
& \quad: J_{1}+J_{2} .
\end{aligned}
$$

By using the triangle inequality and the fact that $t_{0} \in[a, 1]^{N}$, we derive directly that

$$
\begin{aligned}
J_{1} & \leq c \sum_{j=1}^{N} \int_{\left|\xi_{j}\right|^{H_{j}} \leq d_{n-1}}\left(1-\cos \left(\varepsilon_{j}^{(n)} \xi_{j}\right)\right) \frac{d \xi_{j}}{\left|\xi_{j}\right|^{2 H_{j}+1}} \\
& \leq c U^{-2\left(1-H_{N}\right)} \rho\left(0,\left\langle\varepsilon_{j}^{(n)}\right\rangle\right)^{2} .
\end{aligned}
$$

To bound $J_{2}$, notice that $\rho(0, \xi)>d_{n}$ implies $\left|\xi_{j_{0}}\right|^{H_{j_{0}}}>d_{n} / N$ for some $j_{0} \in$ $\{1, \ldots, N\}$. For simplicity of notation, we assume $j_{0}=1$. Then

$$
J_{2} \leq c \int_{\left|\xi_{1}\right|^{H_{1}}>d_{n} / N} \frac{d \xi_{1}}{\left|\xi_{1}\right|^{2 H_{1}+1}}=c d_{n}^{-2} .
$$

Combining (6.6), (6.7), and (6.8), we obtain

$$
\mathbb{E}\left(\widetilde{B}_{n}^{H}\left(t_{0}+\left\langle\varepsilon_{j}^{(n)}\right\rangle\right)-\widetilde{B}_{n}^{H}\left(t_{0}\right)\right)^{2} \leq c \rho\left(0,\left\langle\varepsilon_{j}^{(n)}\right\rangle\right)^{2} \exp \left(-2\left(1-H_{N}\right) \mu n^{\mu}\right),
$$

which is the same as (5.20). Now the same proof as that of Theorem 5.1 shows that for every $t \in[a, 1]^{N}$,

$$
\limsup _{n \rightarrow \infty} \frac{\left|B^{H}\left(t_{0}+\left\langle\varepsilon_{j}^{(n)}\right\rangle\right)-B^{H}\left(t_{0}\right)\right|}{\rho\left(0,\left\langle\varepsilon_{j}^{(n)}\right\rangle\right) \sqrt{\log \log \left(1+\prod_{j=1}^{N}\left|\varepsilon_{j}^{(n)}\right|-H_{j}\right)}} \geq c_{6,1} \quad \text { a.s. }
$$

This proves the lower bounds in (6.3) and 6.4). Finally, by using Lemma 7.1.1 of Marcus and Rosen [20] (one may also apply the wavelet expansion for $B^{H}$ in Ayache and Xiao [2] and Kolmorogov's 0-1 law), we derive from the above that (6.3) and (6.4) hold. 


\subsection{Applications to the solutions of stochastic partial differential equa-}

tions. Gaussian random fields arise naturally as solutions to stochastic partial differential equations. In this subsection, as applications of our results, we establish the global and local moduli of continuity of the solutions of the stochastic heat equation.

Funaki's model for random string in $\mathbb{R}$ is specified by the stochastic heat equation

$$
\frac{\partial u(t, x)}{\partial t}=\frac{\partial^{2} u(t, x)}{\partial x^{2}}+\dot{W}
$$

where $\dot{W}(t, x)$ is an $\mathbb{R}$-valued space-time white noise, which is assumed to be adapted with respect to a filtered probability space $\left(\Omega, \mathcal{F}, \mathcal{F}_{t}, \mathbb{P}\right)$, where $\mathcal{F}$ is complete and the filtration $\left\{\mathcal{F}_{t}, t \geq 0\right\}$ is right continuous; see Funaki [14] and Mueller and Tribe 22 for more information. Recall from Mueller and Tribe 22 that a solution of (6.9) is defined as an $\mathcal{F}_{t}$-adapted, continuous random field $\{u(t, x), t \in$ $\left.\mathbb{R}_{+}, x \in \mathbb{R}\right\}$ with values in $\mathbb{R}$ satisfying the following properties:

(i) $u(0, \cdot) \in \mathcal{E}_{\exp }$ almost surely and is adapted to $\mathcal{F}_{0}$, where $\mathcal{E}_{\exp }=\bigcup_{\lambda>0} \mathcal{E}_{\lambda}$ and

$$
\mathcal{E}_{\lambda}=\left\{f \in C(\mathbb{R}):|f(x)| e^{-\lambda|x|} \rightarrow 0 \text { as }|x| \rightarrow \infty\right\} .
$$

(ii) For every $t>0$, there exists $\lambda>0$ such that $u(s, \cdot) \in \mathcal{E}_{\lambda}$ for all $s \leq t$, almost surely.

(iii) For every $t>0$ and $x \in \mathbb{R}$, the Green's function representation

$$
u(t, x)=\int_{\mathbb{R}} G(t, x-y) u(0, y) d y+\int_{0}^{t} G(t-r, x-y) W(d y d r),
$$

holds, where $G(t, x)=\frac{1}{\sqrt{4 \pi t}} e^{-\frac{x^{2}}{4 t}}$ is the fundamental solution of the heat equation.

We call each solution $\left\{u(t, x), t \in \mathbb{R}_{+}, x \in \mathbb{R}\right\}$ of (6.9) a random string process with values in $\mathbb{R}$, or simply a random string as in Mueller and Tribe [22. Note that in general, a random string may not be Gaussian. A powerful step in the proofs of Mueller and Tribe [22] is to reduce the problems about a general random string process to those of the stationary pinned string $U_{0}=\left\{U_{0}(t, x), t \in \mathbb{R}_{+}, x \in \mathbb{R}\right\}$, obtained by taking the initial function $u(0, \cdot)$ in (6.10) to be defined by

$$
u(0, x)=\int_{0}^{\infty} \int_{\mathbb{R}}(G(r, x-z)-G(r, z)) \widetilde{W}(d z d r),
$$

where $\widetilde{W}$ is a space-time white noise independent of the white noise $\dot{W}$. Consequently, the stationary pinned string is a continuous version of the Gaussian field

$$
\begin{gathered}
U_{0}(t, x)=\int_{0}^{\infty} \int_{\mathbb{R}}(G(t+r, x-z)-G(t+r, z)) \widetilde{W}(d z d r) \\
+\int_{0}^{t} \int_{\mathbb{R}} G(r, x-z) W(d z d r) .
\end{gathered}
$$

Mueller and Tribe [22 proved that the Gaussian field $U_{0}=\left\{U_{0}(t, x), t \in \mathbb{R}_{+}, x \in\right.$ $\mathbb{R}\}$ has stationary increments and satisfies the condition (A1) (with $H_{1}=1 / 2$ and $\left.H_{2}=1\right)$. Let $U_{1}, \ldots, U_{d}$ be independent copies of $U_{0}$, and consider the Gaussian random field $U=\left\{U(t, x), t \in \mathbb{R}_{+}, x \in \mathbb{R}\right\}$ with values in $\mathbb{R}^{d}$ defined by $U(t, x)=\left(U_{1}(t, x), \ldots, U_{d}(t, x)\right)$. Mueller and Tribe 22 found necessary and sufficient conditions (in terms of the dimension $d$ ) for $U$ to hit points or to have double 
points of various types. They have also studied the question of recurrence and transience for $\left\{U(t, x), t \in \mathbb{R}_{+}, x \in \mathbb{R}\right\}$. Wu and Xiao 30. studied the fractal properties of various random sets generated by the random string processes. Further results on hitting probabilities of random string process can be found in Dalang et al. [10. In this subsection we establish the following law of the iterated logarithm for the Gaussian random field $U_{0}$.

Theorem 6.4. Let $\mathbf{t}_{0} \in[a, 1]^{2} \subset \mathbb{R}^{N}$ with $0 \leq a \leq 1$. Then

$$
\lim _{\|\varepsilon\| \rightarrow 0+} \sup _{(|t|,|x|) \leq\left\langle\varepsilon_{j}\right\rangle} \frac{\left|U_{0}\left(\mathbf{t}_{0}+(t, x)\right)-U_{0}\left(\mathbf{t}_{0}\right)\right|}{\gamma\left(\mathbf{t}_{0}, \mathbf{t}_{0}+(t, x)\right)}=\kappa_{7} \quad \text { a.s. },
$$

where $\gamma(s, t)$ is defined as in Theorem 5.1, and

$$
\lim _{\varepsilon \rightarrow 0+} \sup _{s: \sigma(s) \leq \varepsilon} \frac{\left|U_{0}\left(\mathbf{t}_{0}+s\right)-U_{0}\left(\mathbf{t}_{0}\right)\right|}{\sigma(s) \sqrt{\log \log \left(1+\sigma(s)^{-1}\right)}}=\kappa_{8} \quad \text { a.s. }
$$

Here, $\kappa_{7}$ and $\kappa_{8}$ are positive and finite constants.

On the other hand, Robeva and Pitt [27, Proposition 10] showed that the Gaussian random field

$$
u_{0}(t, x)=\frac{1}{2 \pi} \int_{\mathbb{R}^{2}} \frac{e^{i\left(\xi_{1} t+\xi_{2} x\right)}-1}{i \xi_{1}+\xi_{2}^{2}} \widetilde{\mathcal{M}}\left(d \xi_{1}, d \xi_{2}\right), \quad \forall t \in \mathbb{R}_{+}, x \in \mathbb{R},
$$

is another solution to (6.9) satisfying $u_{0}(0,0)=0$. Here $\widetilde{\mathcal{M}}$ is a complex Gaussian white noise in $\mathbb{R}^{2}$. This Gaussian random field has stationary increments with spectral density

$$
f(\xi)=\frac{1}{\xi_{1}^{2}+\xi_{2}^{4}}
$$

Hence, one can verify that the Gaussian random field $\left\{u_{0}(t, x), t \in \mathbb{R}_{+}, x \in \mathbb{R}\right\}$ satisfies conditions (A1) (with $H_{1}=1 / 4$ and $H_{2}=1 / 2$ ) and (A3). Therefore, as applications of Theorems 4.1 and 5.1, we establish the following global and local moduli of continuity for the Gaussian field $\left\{u_{0}(t, x), t \in \mathbb{R}_{+}, x \in \mathbb{R}\right\}$.

Theorem 6.5. We have

$$
\lim _{\varepsilon \rightarrow 0+} \sup _{\substack{\left(t_{1}, x_{1}\right),\left(t_{2}, x_{2}\right) \in I \\ \sigma\left(\left(t_{1}, x_{1}\right),\left(t_{2}, x_{2}\right)\right) \leq \varepsilon}} \frac{\left|u_{0}\left(t_{1}, x_{1}\right)-u_{0}\left(t_{2}, x_{2}\right)\right|}{\beta\left(\left(t_{1}, x_{1}\right),\left(t_{2}, x_{2}\right)\right)}=\kappa_{9} \quad \text { a.s. }
$$

where $\beta(\cdot, \cdot)$ is defined as in (4.1) and $\kappa_{9}$ is a positive constant satisfying (4.3) (with $H_{1}=1 / 4$ and $\left.H_{2}=1 / 2\right)$.

Theorem 6.6. Let $\mathbf{t}_{0} \in[a, 1]^{2} \subset \mathbb{R}^{N}$ with $0 \leq a \leq 1$. Then, there exist positive and finite constants $\kappa_{10}$ and $\kappa_{11}$ such that

$$
\lim _{\|\varepsilon\| \rightarrow 0+} \sup _{(|t|,|x|) \leq\left\langle\varepsilon_{j}\right\rangle} \frac{\left|u_{0}\left(\mathbf{t}_{0}+(t, x)\right)-u_{0}\left(\mathbf{t}_{0}\right)\right|}{\gamma\left(\mathbf{t}_{0}, \mathbf{t}_{0}+(t, x)\right)}=\kappa_{10} \quad \text { a.s. }
$$

where $\gamma(s, t)$ is defined as in Theorem 5.1, and

$$
\lim _{\varepsilon \rightarrow 0+} \sup _{s: \sigma(s) \leq \varepsilon} \frac{\left|u_{0}\left(\mathbf{t}_{0}+s\right)-u_{0}\left(\mathbf{t}_{0}\right)\right|}{\sigma(s) \sqrt{\log \log \left(1+\sigma(s)^{-1}\right)}}=\kappa_{11} \quad \text { a.s. }
$$

\section{ACKNOWLEDGEMENTS}

The authors wish to express their deep gratitude to a referee for his/her valuable comments on an earlier version which improved the quality of this paper. 


\section{REFERENCES}

[1] T. W. Anderson (1955), The integral of a symmetric convex set and some probability inequalities. Proc. Amer. Math. Soc. 6, 170-176. MR0069229 (16:1005a)

[2] A. Ayache and Y. Xiao (2005), Asymptotic properties and Hausdorff dimensions of fractional Brownian sheets. J. Fourier Anal. Appl. 11, 407-439. MR2169474 (2006g:60056)

[3] A. Benassi, S. Jaffard and D. Roux (1997), Elliptic Gaussian random processes. Rev. Mat. Iberoamericana 13, 19-90. MR 1462329 (98k:60056)

[4] D. A. Benson, M. M. Meerschaert, B. Baeumer and H. P. Scheffler (2006), Aquifer operatorscaling and the effect on solute mixing and dispersion. Water Resources Research 42, no. 1, W01415 (18pp.).

[5] S. M. Berman (1985), An asymptotic bound for the tail of the distribution of the maximum of a Gaussian process with stationary increments. Ann. Inst. Henri. Poincaré Probab. Statist 21, 47-57. MR.791269 (86k:60063)

[6] H. Biermé, M. M. Meerschaert and H.-P. Scheffler (2007), Operator scaling stable random fields. Stoch. Process. Appl. 117, 312-332. MR2290879 (2007k:60146)

[7] A. Bonami and A. Estrade (2003), Anisotropic analysis of some Gaussian models. J. Fourier Anal. Appl. 9, 215-236. MR1988750 (2004e:60082)

[8] E. Csáki, M. Csörgő and Q.-M. Shao (1992), Fernique type inequalities and moduli of continuity for $l^{2}$-valued Ornstein-Uhlenbeck processes. Ann. Inst. Henri. Poincaré Probab. Statist. 28, 479-517. MR 1193082 (93k:60095)

[9] R. C. Dalang (1999), Extending martingale measure stochastic integral with applications to spatially homogeneous s.p.d.e.'s. Electron. J. Probab. 4, no.6, 1-29. Erratum in Electron. J. Probab. 6 (2001), No.6, 1-5. MR.1684157 (2000b:60132)

[10] R. C. Dalang, C. Mueller and L. Zambotti (2006), Hitting properties of parabolic s.p.d.e.'s with reflection. Ann. Probab. 34, 1423-1450. MR2257651 (2007e:60054)

[11] S. Davies and P. Hall (1999), Fractal analysis of surface roughness by using spatial data (with discussion). J. Roy. Statist. Soc. Ser. B 61, 3-37. MR 1664088 (99i:62124)

[12] N. Eisenbaum and D. Khoshnevisan (2002), On the most visited sites of symmetric Markov processes. Stoch. Process. Appl. 101, 241-256. MR1931268 (2003i:60122)

[13] X. Fernique (1975), Régularité des Trajectoires des Fonctions Aléatoires Gaussiennes. In: École d'été des Probabilités de St-Flour, IV-1974. Lect. Notes Math. 480, pp. 1-96. SpringerVerlag, Berlin. MR0413238 (54:1355)

[14] T. Funaki (1983), Random motion of strings and related stochastic evolution equations. Nagoya Math. J. 89, 129-193. MR692348(85g:60063)

[15] A. Kamont (1996), On the fractional anisotropic Wiener field. Probab. Math. Stat. 16, 85-98. MR.1407935(98a:60064)

[16] D. Khoshnevisan and Y. Xiao (2007), Images of the Brownian sheet. Trans Amer. Math. Soc. 359, 3125-3151. MR2299449 (2008g:60107)

[17] W. V. Li and Q.-M. Shao (2001), Gaussian processes: inequalities, small ball probabilities and applications. In: Stochastic Processes: Theory and Methods, Handbook of Statistics 19 (C. R. Rao and D. Shanbhag, editors), pp. 533-597, North-Holland. MR.1861734

[18] M, Loève (1977), Probability Theory I. Springer-Verlag, New York. MR0651017 (58:31324a)

[19] N. Luan and Y. Xiao (2010). Exact Hausdorff measure functions for the trajectories of anisotropic Gaussian random fields. Preprint.

[20] M. B. Marcus and J. Rosen (2006), Markov Processes, Gaussian Processes, and Local Times. Cambridge University Press, Cambridge. MR 2250510 (2008b:60001)

[21] D. Monrad and H. Rootzén (1995), Small values of Gaussian processes and functional laws of the iterated logarithm. Probab. Th. Rel. Fields 101, 173-192. MR1318191 (96a:60032)

[22] C. Mueller and R. Tribe (2002), Hitting probabilities of a random string. Eletron. J. Probab. 7, No. 10, 1-29. MR1902843(2003g:60111)

[23] D. Nualart (2006), Stochastic heat equation driven by fractional noise. Preprint.

[24] B. Øksendal and T. Zhang (2000), Multiparameter fractional Brownian motion and quasilinear stochastic partial differential equations. Stoch. Stoch. Rep. 71, 141-163. MR.1922562 (2003k:60174)

[25] S. Orey and W. E. Pruitt (1973), Sample functions of the $N$-parameter Wiener process. Ann. Probab. 1, 138-163. MR0346925 (49:11646) 
[26] L. D. Pitt (1978), Local times for Gaussian vector fields. Indiana Univ. Math. J. 27, 309-330. MR0471055(57:10796)

[27] R. S. Robeva and L. D. Pitt (2004), On the equality of sharp and germ $\sigma$-fields for Gaussian processes and fields. Pliska Stud. Math. Bulgar. 16, 183-205. MR2070315(2005e:60083)

[28] M. Talagrand (1995), Hausdorff measure of trajectories of multiparameter fractional Brownian motion. Ann. Probab. 23, 767-775. MR.1334170 (96f:60067)

[29] W. Wang (2007), Almost-sure path properties of fractional Brownian sheet. Ann. Inst. Henri Poincaré Probab. Statist. 43, 619-631. MR2347099(2008j:60092)

[30] D. Wu and Y. Xiao (2007), Geometric properties of fractional Brownian sheets. J. Fourier Anal. Appl. 13, 1-37. MR2296726 (2008b:60078)

[31] Y. Xiao (1996), Hausdorff measure of the sample paths of Gaussian random fields. Osaka J. Math. 33, 895-913. MR1435460 (98c:60045)

[32] Y. Xiao (2009), Sample path properties of anisotropic Gaussian random fields. In: A Minicourse on Stochastic Partial Differential Equations, D. Khoshnevisan, F. Rassoul-Agha, editors, Lecture Notes in Math. 1962, pp 145-212, Springer, New York. MR2508776 (2010i:60159)

[33] A. M. Yaglom (1957), Some classes of random fields in $n$-dimensional space, related to stationary random processes. Th. Probab. Appl. 2, 273-320. MR0094844 (20:1353)

Department of Statistics and Probability, Michigan State University, A-413 Wells Hall, East Lansing, Michigan 48824

E-mail address: mcubed@stt.msu.edu

Department of Mathematics, Hangzhou Normal University, Hangzhou, 310036, PeoPLE'S REPUBLIC OF CHINA

E-mail address: wswang@stat.ecnu.edu.cn

Department of Statistics and Probability, Michigan State University, A-413 Wells Hall, East Lansing, Michigan 48824

E-mail address: xiao@stt.msu.edu 